\title{
A Structural Jump Threshold Framework for Credit Risk
}

\author{
Pierre Garreau \\ Department of Mathematics \\ Goethe University \\ Robert-Mayer-Str. 6-10 \\ 60325 Frankfurt \\ garreau@math.uni-frankfurt.de
}

\author{
Alec Kercheval \\ Department of Mathematics \\ Florida State University \\ 1017 Academic Way, room 208 \\ Tallahassee, FL 32306-4510 \\ wWw.math.fsu.edu/ kercheva
}

\begin{abstract}
This paper presents a new structural framework for multidimensional default risk. We define the time of default as the first time the log-return of the stock price of a firm jumps below a (possibly nonconstant) default level. When stock prices are exponential Lévy, this framework is equivalent to a reduced form approach, where the intensity process is parametrized by a Lévy measure. The dependence between the default times of firms within a basket of credit securities is the result of the jump dependence of their respective stock prices, making the link between the equity and credit markets. We value a first-todefault basket CDS as an application.
\end{abstract}

Keywords: Lévy processes, Lévy copula, credit risk, structural models, reduced form models. 


\section{Introduction}

There currently exist two main classes of models for default risk: structural and reduced form models. We refer to $[4,5,15,17,21]$ for the first class of models, and to $[6,11,12,23]$ for the second. Both types of models define a framework for understanding credit risk by modeling the timing of the default of a firm. In the framework of structural models, the default time $\tau$ is the first passage time of the firm's value process below a given threshold. In reduced form models, $\tau$ is an ad hoc function of market observables such as interest rates, which are not directly linked to the firm's fundamentals, with spreads that are estimated statistically.

A structural model has the advantage of a transparent economic interpretation with a direct link to firm values. However, with the gradual abandonment of geometric Brownian motion in favor of jump models for underlying stock prices, structural models generally lack explicit formulas for default probabilities, especially in the multi-dimensional case. Reduced form models tend to enjoy explicit default formulas for more general stock price processes, but are more loosely linked to asset fundamentals. In this paper we present a modeling approach to pricing credit derivatives on firms and portfolios of firms that combines advantages of both structural and reduced form models.

To motivate our approach, consider Figure 1, showing the stock price of MF Global near its bankruptcy. The firm's default occurred not at the largest drop in the firm's value, but later at the largest drop in the firm's return.

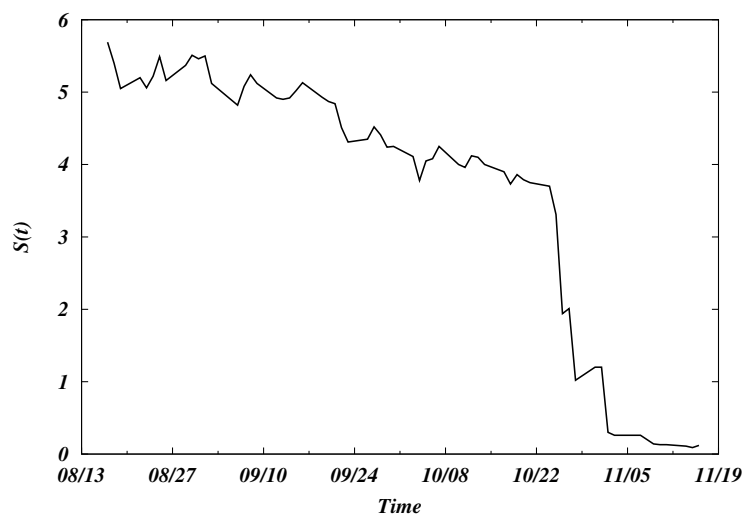

(a) MF Global. Stock price.

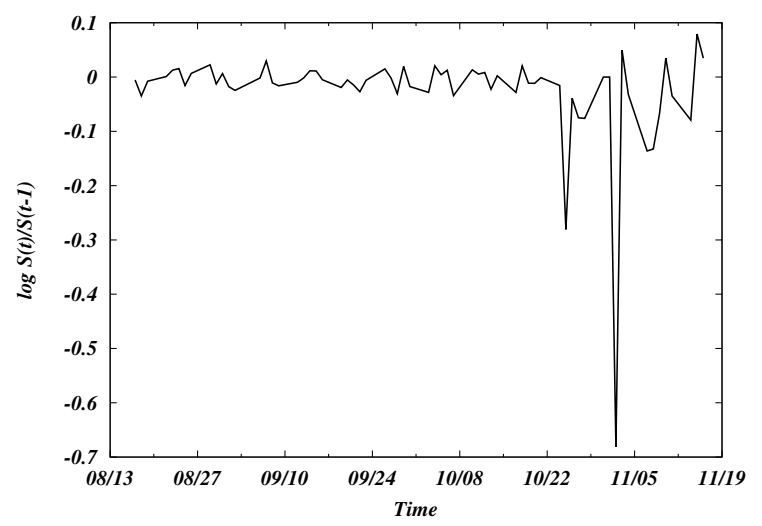

(b) MF Global. Daily log returns.

Figure 1: Bankruptcy of MF Global, October-November 2011. Bankruptcy occurred on Oct. 31, 2011, not at the largest absolute drop in stock price, but at the largest relative drop.

Our approach is the following. We consider rapid changes in the stock price $S_{t}$ as represented by the instantaneous log-return at time $t, \log \left(S_{t} / S_{t^{-}}\right)$. The default time of a firm is modeled as the first time that the log-return of the stock price jumps below a (possibly non-constant) default level. That is, we take the default time $\tau$ to be given by

$$
\tau=\inf \left\{t>0: \log \left(S_{t} / S_{t^{-}}\right) \leq a_{t}\right\}
$$

where $a_{t}$ is a (negative) default threshold and $S_{t}$ is the stock price process. Our analysis requires only that $S_{t}$ is an exponential Lévy process, and yields simple pricing formulas that 
are easy to compute even in the multi-dimensional case without the need for PIDE solvers or Monte Carlo simulation.

For a portfolio of $N$ stocks, we only need to assume that the prices are driven by the components of an $\mathbb{R}^{N}$-valued Lévy process. By means of the use of Lévy copulas, we can decouple the dependence structure of the jumps from the set of individual jump intensities of the firms. As in the work of Marshall and Olkin [19], we restrict attention throughout this paper to the case $N=2$ in order to simplify the analysis and notation, though the results all carry over similarly for general $N$.

For illustration, we price a first-to-default basket credit default swap on two underliers. The formulas are explicit and allow for separate calibration of the individual stock processes and the dependence structure.

Our approach enjoys these advantages:

1. as a structural model it is directly linked to the observable stock price process $S_{t}$. It may be calibrated directly to $S_{t}$, giving us an advantage over typical structural models formulated in terms of a firm value process that is not directly observable;

2. the framework allows for the use of a consistent set of underlying models to price credit derivates and options on the same assets;

3. probabilities and prices are explicit, equivalent to reduced form models, and furthermore the dependence structure enters the model in an explicit way that can be separately calibrated or studied;

4. the method gives explicit solutions for multidimensional problems (e.g. basket CDS pricing);

5. the general results do not depend on any ad hoc price modeling choices, but hold generally for any exponential Lévy stock price models, i.e. only rely on the mild assumption that log-prices jointly have independent and stationary increments. This provides the flexibility to use most of the currently popular price process models, including pure jump processes like Variance Gamma, Normal Inverse Gaussian, CGMY, and tempered stable processes.

Because the instantaneous log-return vanishes except where the process $S_{t}$ jumps, this approach won't work for continuous price models like geometric Brownian motion. This could explain why our approach would not have been obvious or tractable in the early days of asset pricing theory when Brownian motion was the primary innovation. Since then, however, we know that asset returns are not Normal, but require heavy-tailed distributions to describe them [18]. As a result, Lévy models have generally replaced Gaussian models for price and risk models that need to be sensitive to the distribution of extreme events. More recent work on the dependence of Lévy processes, especially the Lévy copula theory of Tankov [25], now make this new framework tractable in a way that it would not have been in the 1960's.

The event of default is determined by the relationship between the log-return of the stock price and the default level $a_{t}$. The modeler may choose to think of the default level as a quantity only accessible to the firm management, or, as in the reduced form framework, as a 
statistical level estimated by credit derivatives. The possibility that the default level might be stochastic provides quite a bit of flexibility for the modeler to adjust the probability of default in relation to the variability of the stock price. In this sense, our jump-default framework shifts attention regarding default events from the absolute level of the stock price to its log-variability, which, in the case of pure jump processes, is wholly measured by the jump distribution.

This jump-default framework has the disadvantage, from a structural perspective, that the default event is only triggered by large downward price jumps. There is no level below which the price can slowly diffuse to trigger default. For constant default thresholds, this means the default framework presented here should be thought of as focused on relatively short term sudden and unpredictable defaults that take the market by surprise. This is consistent with the typical debt collection process of margin calls and debt payments incurred by a firm as a discontinuous and somewhat unpredictable process. It is tempting to imagine that during a slow decline, firms have more time to reorganize their assets and debts to avoid default. On the other hand, a trend or diffusion could be incorporated into the default threshold process $a_{t}$ in order to re-introduce a version of a slow diffusion-related trend in default probability.

This paper is organized as follows. In Section 2 we describe the default model in the onedimensional case and develop the single-name default probability formulas in terms of tail integrals of the log-price process. Section 3 develops our analysis of the dependence structure of jumps of a multi-dimensional Lévy process. We start by characterizing the dependence of jumps of a two dimensional homogeneous Poisson process in terms of the joint distribution of survival times, which is bivariate exponential and always follows a Marshall-Olkin copula. This is true independently of the choice of Lévy process, with parameters depending on the Lévy measures. This result generalizes to two-dimensional Lévy processes in Theorem 3.15.

Section 4 presents the joint survival probability formulas for constant, deterministic, and stochastic default levels. These are applied in Section 5 to the problem of pricing a first-todefault basket CDS, to illustrate the use of the results of Section 4. We emphasize the fact that the dependence structure is decoupled from the single-name default probabilities, and so can be modeled independently. Concluding remarks are in Section 6, and proofs of most of the Propositions and Theorems are collected in Section 7.

Acknowledgement: the authors thank the editors and anonymous referees for helpful comments.

\section{A structural jump-threshold model}

Before developing the dependence ideas needed to describe multi-name default probabilities and basket default prices, we describe our default framework for a single asset. For standard definitions and basic concepts involving random measures, Lévy processes and Lévy measures, we refer the reader to any of a variety of excellent available references, including $[1,2,7,8$, $16,22,24]$.

We assume that the interest rate process $\left(r_{t}\right)_{t>0}$ is a constant $r$. Let $Y_{t}$ be a onedimensional Lévy process on a filtered probability space $\left(\Omega, \mathcal{F}, \mathbb{P},\left(\mathcal{F}_{t}\right)_{t \geq 0}\right)$, denote by boldface i the imaginary unit, and $\psi(u)=\log \mathbb{E}\left[e^{\mathbf{i} u Y_{1}}\right]$ the characteristic exponent of $Y_{1}$. With this notation, we model the movements of the stock price $S$ of a firm as a general exponential 
Lévy process by

$$
S_{t}=s \exp \left\{r t+Y_{t}+t \psi(-\mathbf{i})\right\} .
$$

With this specification, the discounted value of the stock price $\tilde{S}_{t}=S_{t} e^{-r t}$ is an $\left(\mathcal{F}_{t}\right)_{t \geq 0^{-}}$ martingale under $\mathbb{P}$. Recall that a Lévy process $Y$ has an associated Lévy measure $\lambda$, such that, for a Borel measurable set $A \in \mathcal{B}(\mathbb{R} \backslash\{0\}), \lambda(A)$ is the expected number of jumps of $Y$ per unit time with jump size $Y_{t}-Y_{t^{-}} \in A$.

The price process $S_{t}$ is the solution of the stochastic differential equation

$$
d S_{t}=S_{t^{-}}\left(r d t+\sigma d W_{t}+\int_{\mathbb{R}}\left(e^{x}-1\right)\{X(d t \times d x)-d t \times \lambda(d x)\}\right), \quad S_{0}=s>0,
$$

where $X$ is the Poisson random measure on $[0, \infty) \times(\mathbb{R} \backslash\{0\})$ with intensity $L e b \times \lambda$ associated to $Y$ via the Levy-Itô decomposition. (Leb denotes Lebesgue measure.) We call $X$ a temporal Poisson random measure (TPRM) on $\mathbb{R} \backslash\{0\}$ with intensity $\lambda$.

An important quantity associated to $Y$ is the tail integral of the process $Y$, defined to be the function $\Lambda(x)=\int_{(-\infty, x]} \lambda(d w)$, for $x<0$. This can be interpreted as the intensity of a Poisson process that jumps whenever $Y$ jumps downward by more than $-x$, i.e. $Y_{t}-Y_{t^{-}} \leq x$. (See Section 3.2 for more details.)

Since Brownian motion is a Lévy process, we can think of this stock price model as a natural generalization of the geometric Brownian model

$$
d S_{t}=S_{t}\left(r d t+\sigma d W_{t}\right)
$$

to a much wider class of processes that includes jump diffusions and many popular pure jump processes [8].

Given a firm with stock price $S$, the event of default is defined as the first time the log return of the stock price jumps below a default threshold level $a<0$, where a can in general be non-constant and non-deterministic. More precisely:

Definition 2.1 (Default time, default level process). Given the exponential Lévy stock price $S_{t}$ defined above, a default level process is a real-valued $\mathcal{F}_{t}$-progressively measurable random process $\left\{a_{t}\right\}$ uniformly bounded below zero. The corresponding default time $\tau$ is defined as

$$
\tau(\omega)=\inf \left\{t>0: \log \left(S_{t}(\omega) / S_{t^{-}}(\omega)\right) \leq a_{t}(\omega)\right\} .
$$

The default level $a_{t}$ represents the percentage value of a shock observed in the stock price at time $t$ needed to trigger default. In many cases $a_{t}$ can be deterministic or constant.

In order to price credit derivatives on $S$, we need to evaluate the survival probabilities, described next.

Proposition 2.2 (Default probability, hazard rate, stochastic default level). Suppose the stock price $S_{t}$ is an exponential Lévy process and $a_{t}$ is a predictable default level process independent of $S_{t}$.

Then

1. the survival probability is given by

$$
\mathbb{P}(\tau>t)=\mathbb{E}\left[\exp \left\{-\int_{0}^{t} \Lambda\left(a_{u}\right) d u\right\}\right]
$$

where $\Lambda(a)=\int_{(-\infty, a]} \lambda(d w)$ is the tail integral of the process $Y$. 
2. the hazard rate (also sometimes called the instantaneous default rate or local default rate) $H_{t}$ is, for a.e. $t$,

$$
H_{t} \equiv \lim _{h \downarrow 0} \frac{\mathbb{P}(\tau \leq t+h \mid \tau>t)}{h}=\mathbb{E}\left[\Lambda\left(a_{t}\right) \mid \tau>t\right]=\frac{\mathbb{E}\left[\Lambda\left(a_{t}\right) e^{-\int_{0}^{t} \Lambda\left(a_{s}\right) d s}\right]}{\mathbb{E}\left[e^{-\int_{0}^{t} \Lambda\left(a_{s}\right) d s}\right]} .
$$

An important special case for applications is the case where the default level $a_{t}$ is a deterministic function of time (for example, piecewise constant, as in [11]).

Corollary 2.3 (Default probability, hazard rate, deterministic default level). Let the stock price $S$ of a firm be an exponential Lévy process (1) and suppose the default level $a_{t}$ is deterministic. The survival probability up to time $t>0$ is given by

$$
\mathbb{P}(\tau>t)=\exp \left\{-\int_{0}^{t} \Lambda\left(a_{u}\right) d u\right\}
$$

Furthermore, for almost every $t \geq 0$, the hazard rate $H_{t}$ exists and is given by

$$
H_{t} \equiv \lim _{h \downarrow 0} \frac{\mathbb{P}(\tau \leq t+h \mid \tau>t)}{h}=\Lambda\left(a_{t}\right) .
$$

If in addition the default level $a_{t}$ is right continuous and the Lévy measure $\lambda$ of $Y$ is nonatomic, then equation (7) holds for all $t \geq 0$.

If the default threshold level $a_{t}$ is constant, $a_{t}=a$, then we obtain the even simpler formulas

$$
\mathbb{P}(\tau>t)=e^{-t \Lambda(a)}
$$

and

$$
H_{t} \equiv \lim _{h \downarrow 0} \frac{\mathbb{P}(\tau \leq t+h \mid \tau>t)}{h}=\Lambda(a) .
$$

We notice that the typical formulas of reduced form models are appearing here in this structural context. In this simplest case of constant default level, the default time is exponential, and the average waiting time to default, $\Lambda(a)$, is parametrized by the Lévy measure of the underlying process. The reason for this is that the "tail process" $N_{t}$ marking jumps of $Y_{t}$ beyond the default threshold is a homogeneous Poisson process with mean $\Lambda(a)$.

To be more specific, we write $E=\mathbb{R} \backslash\{0\}$ and $I(z, A)$ to be the indicator function with value 1 if $z \in A$ and value 0 otherwise. If we define

$$
N_{t}(\omega)=\int_{[0, t] \times E} I(z,(-\infty, a]) X(\omega, d s \times d z),
$$

then $N$ is a homogeneous Poisson process. The proof of the non-constant case proceeds by establishing that the tail process

$$
N_{t}(\omega)=\int_{[0, t] \times E} I\left(z,\left(-\infty, a_{s}\right]\right) X(\omega, d s \times d z) .
$$

is a non-stationary Poisson process with intensity $\left(\Lambda\left(a_{t}\right)\right)_{t \geq 0}$. (All proofs appear in Section 7.) 
Example 2.4. Assume that the dynamics of the the stock price $S$ of a firm is given by (1), where $\lambda$ is the Lévy measure of an $\alpha$-stable process,

$$
\lambda(d x)=\alpha\left(\frac{c_{+}}{x^{1+\alpha}} 1_{x>0}+\frac{c_{-}}{|x|^{1+\alpha}} 1_{x<0}\right)
$$

$\alpha \in(0,1) \cup(1,2), c_{-}, c_{+}>0$. Then the tail integral of $\lambda$ on the negative axis becomes

$$
\forall x \in(-\infty, 0), \quad \Lambda(x)=\frac{c_{-}}{|x|^{\alpha}} .
$$

Therefore the survival probability for a constant default level a is

$$
\mathbb{P}(\tau>t)=e^{-t c_{-} /|a|^{\alpha}} .
$$

The two following examples show how one can construct models with nonconstant deterministic intensities.

Example 2.5 (Piecewise constant default level). Let $0=t_{0}<t_{1}<\ldots<t_{I}$ and define the default level a such that

$$
\forall t \geq 0, \quad a_{t}=\sum_{i=1}^{I} a_{i} 1_{\left[t_{i-1}, t_{i}\right)}(t)+a_{I} 1_{t \geq t_{I}},
$$

where $a_{i}<0, i=1, \ldots, I$. For $t \in\left[t_{k}, t_{k+1}\right), k=1, \ldots, I-1$, the survival probability up to time $t$ is

$$
\mathbb{P}(\tau>t)=\exp \left\{-\sum_{i=1}^{k} \Lambda\left(a_{i}\right)\left(t_{i}-t_{i-1}\right)-\Lambda\left(a_{k}\right)\left(t-t_{k}\right)\right\} .
$$

Example 2.6 (Linear default level, $\alpha$-stable process). Assume that the Lévy measure of $Y$ is as in Example 2.4 with $\alpha \in(1,2)$ and that the default level is represented by the function $a: t \rightarrow a_{t}=\min (\beta t-\gamma,-\epsilon)$ for $\epsilon>0$. Then, for $t<(\gamma-\epsilon) / \beta$, the hazard rate $\left(H_{t}\right)_{t \geq 0}$ is

$$
H_{t}=\Lambda\left(a_{t}\right)=\frac{c_{-}}{|\beta t-\gamma|^{\alpha}}
$$

and the survival probability is

$$
\mathbb{P}(\tau>t)=\exp \left\{\frac{c_{-}}{\beta(\alpha-1)}\left[(\gamma-\beta t)^{1-\alpha}-\gamma^{1-\alpha}\right]\right\} .
$$

We plot the default level $\left(a_{t}\right)_{t \geq 0}$ for the piecewise constant and linear models of Examples 2.5 and 2.6 in Figure 2.

Further discussion, and examples with stochastic default levels, may be found in [9]. We emphasize that our results for stochastic default levels are limited to the case when the default level process $a_{t}$ is assumed independent of the stock price process $S_{t}$. When this is the case, the extension from the deterministic to the stochastic case is straightforward. The hazard rates are included in the statements above to add some intuition to the results, but we only need the survival probabilities to compute CDS prices in later sections. 


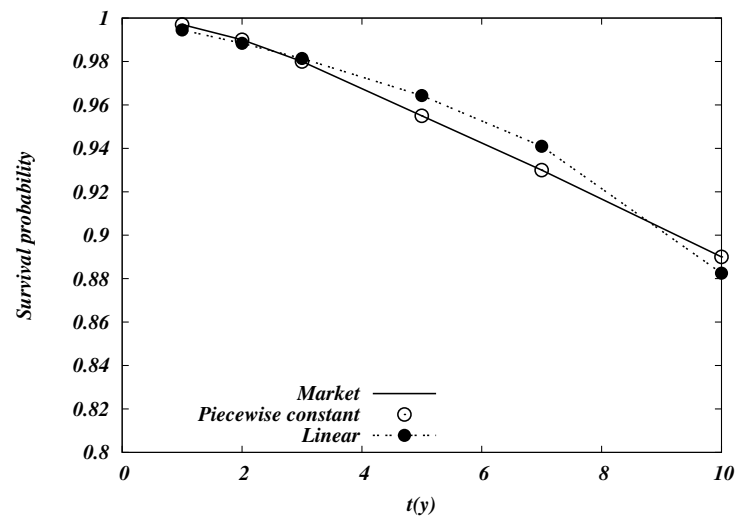

(a) Survival probability $\mathbb{P}(\tau>t)$.

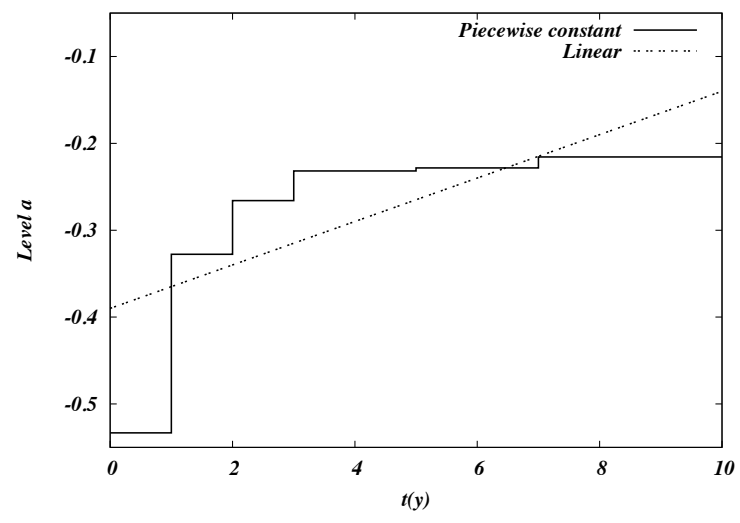

(b) Default level $a_{t}$.

Figure 2: Survival probabilities (left) and default level $\left(a_{t}\right)_{t \geq 0}$ (right) in a jump threshold model with deterministic default level and $\alpha$-stable Lévy measure. $\alpha=3 / 2, c_{-}=1 / 100$. Market value taken from [24].

\section{Jump dependence for Lévy processes}

We need to develop a few ideas and notations for the dependence of jumps of Lévy processes in this section, in terms of which the default pricing formulas will be expressed later. We follow some of the literature and choose to simplify notation by restricting attention to two dimensions. However, nothing changes except notational complexity in considering higher dimensional dependence.

\subsection{Two-dimensional homogeneous Poisson processes}

It's helpful to start with the special case of homogeneous Poisson processes, and then move to general Lévy processes. Here the term "Poisson process", with no other modifiers, will always mean a classical one-dimensional homogeneous Poisson process on $\mathbb{R}^{+}$.

Because the survival times of a Poisson process are exponential random variables, we need to understand univariate and bivariate exponential variables.

First we recall the two-dimensional version of the memoryless property as developed by Marshall and Olkin [19].

Definition 3.1 ([19], Two-dimensional memoryless property). A pair of non-negative random variables $(S, T)$ has the memoryless property if

$$
\forall s_{1}, s_{2}, t \geq 0, \mathbb{P}\left(S>s_{1}+t, T>s_{2}+t \mid S>t, T>t\right)=\mathbb{P}\left(S>s_{1}, T>s_{2}\right) .
$$

Marshall and Olkin showed that the only pair of random variables $(S, T)$ which satisfies 3.1 is the bivariate exponential random vector.

Definition 3.2 ([19], Bivariate exponential random vector). A pair of random variables $S$ and $T$ forms a Bivariate exponential random vector if there exist $\lambda, \mu, \rho \geq 0$, with $\rho \in[0, \min (\lambda, \mu)]$, such that

$$
\forall s, t \geq 0, \quad \mathbb{P}(S>s, T>t)=e^{-(\lambda-\rho) s-(\mu-\rho) t-\rho \max (s, t)} .
$$

We write $(S, T) \sim b \mathcal{E}(\lambda, \mu, \rho)$. 
If $(S, T) \sim b \mathcal{E}(\lambda, \mu, \rho)$ it follows that $S$ and $T$ are exponential with parameters $\lambda$ and $\mu$, respectively, written $S \sim \mathcal{E}(\lambda)$ and $T \sim \mathcal{E}(\mu)$.

We can understand the bivariate exponentials in terms of exponential marginals and the classical notion of copulas (e.g. Nelsen [20], to be distinguished from the Lévy copulas described below). Given two one dimensional exponential random variables $S$ and $T$ with respective parameters $\lambda$ and $\mu$, it is a remarkable fact that the only copula making $(S, T)$ a bivariate exponential random vector is the three parameter Marshall-Olkin copula.

Definition 3.3 (Three parameter Marshall-Olkin copula). Let $\lambda, \mu \in \mathbb{R}_{+}$and $\rho \in[0, \min (\lambda, \mu)]$. The three parameter Marshall-Olkin copula is the function $C_{\rho}^{\lambda, \mu}:[0,1]^{2} \rightarrow[0,1]$ defined by

$$
C_{\rho}^{\lambda, \mu}(u, v)=u v \min \left(u^{-\rho / \lambda}, v^{-\rho / \mu}\right) .
$$

The claims above are included in the following summarizing statement.

Theorem 3.4 ([19], [20], Characterization of a bivariate exponential). Let $S, T$ be random variables on a probability space $(\Omega, \mathcal{F}, \mathbb{P})$. The following are equivalent:

(i) $(S, T)$ satisfies the two-dimensional memoryless property.

(ii) $(S, T)$ is a bivariate exponential random vector.

(iii) $S$ and $T$ are exponential and there exists three independent exponential random variables $U, V, W$ such that $S=\min (U, W)$ and $T=\min (V, W)$.

(iv) There exists $\lambda, \mu \geq 0$, and $0 \leq \rho \leq \min (\lambda, \mu)$ such that

$$
\forall s, t \geq 0, \quad \mathbb{P}(S>s, T>t)=C_{\rho}^{\lambda, \mu}\left(e^{-\lambda s}, e^{-\mu t}\right) .
$$

Condition (iv) above immediately implies that $S$ and $T$ are exponential $\mathcal{E}(\lambda)$ and $\mathcal{E}(\mu)$, respectively. A simple fact about exponential random variables is that if $Y_{1} \sim \mathcal{E}\left(\mu_{1}\right)$ and $Y_{2} \sim \mathcal{E}\left(\mu_{2}\right)$ are independent, then $Z=\min \left(Y_{1}, Y_{2}\right) \sim \mathcal{E}\left(\mu_{1}+\mu_{2}\right)$. Hence, in case the conditions of Theorem 3.4 hold, we can take $U \sim \mathcal{E}(\lambda-\rho), V \sim \mathcal{E}(\mu-\rho)$, and $W \sim \mathcal{E}(\rho)$.

We now are interested in two-dimensional processes $Y=(N, M)$, where $N$ and $M$ are one-dimensional Poisson processes. It is not difficult to see that not all such processes $Y$ need be two-dimensional Lévy processes, because the jumps of $N$ could depend on past values of $M$, for example. We therefore introduce the following definition.

Definition 3.5 (Two dimensional Poisson process). A two dimensional Poisson process $Y=$ $(N, M)$ is a two dimensional Lévy process where $N$ and $M$ are one dimensional Poisson processes.

Definition 3.6 (Joint survival times of a two dimensional process with Poisson margins). Let $N, M$ be two Poisson processes on $\left(\Omega, \mathcal{F}, \mathbb{P},\left(\mathcal{F}_{t}\right)_{t \geq 0}\right)$. Define for $u \in[0, \infty)$,

$$
\tau_{u}=\inf \left\{t \geq 0: N_{t+u}>N_{u}\right\}, \quad \text { and } \quad \gamma_{u}=\inf \left\{t \geq 0: M_{t+u}>M_{u}\right\} .
$$

The collection $\left\{\left(\tau_{u}, \gamma_{u}\right), u \geq 0\right\}$ is called the collection of joint survival times of the two dimensional process $(N, M)$. 
The following theorem summarizes the main properties of two dimensional Poisson processes we need in the next section. See also [8] for a related discussion of the decomposition facts.

Theorem 3.7. Let $N$ and $M$ be two Poisson processes on $\left.\left(\Omega, \mathcal{F}, \mathbb{P},\left(\mathcal{F}_{t}\right)_{t \geq 0}\right)\right)$ with parameters $\lambda$ and $\mu$. Suppose $(N, M)$ is a two dimensional Poisson process.

Then

(i) - there exists $\rho \leq \min (\lambda, \mu)$, such that every pair of the collection $\left\{\left(\tau_{u}, \gamma_{u}\right), u \geq 0\right\}$ of joint survival times of $N$ and $M$ is $b \mathcal{E}(\lambda, \mu, \rho)$, and for all $s, t>0$,

$$
\mathbb{P}\left(\tau_{u}>s, \gamma_{u}>t\right)=C_{\rho}^{\lambda, \mu}\left(e^{-\lambda s}, e^{-\mu t}\right),
$$

and

(ii) - There exists three independent adapted Poisson processes $N^{\perp}, M^{\perp}$ and $L$ on $\left(\Omega, \mathcal{F}, \mathbb{P},\left\{\mathcal{F}_{t}\right\}_{t \geq 0}\right)$, with respective parameters $\lambda-\rho, \mu-\rho$ and $\rho$, such that the decomposition (25) holds:

$$
\begin{aligned}
N_{t} & =N_{t}^{\perp}+L_{t} \\
M_{t} & =M_{t}^{\perp}+L_{t} .
\end{aligned}
$$

It follows immediately that for any two-dimensional Poisson process $(N, M)$, either $N$ and $M$ are independent, or there is a positive probability that $N$ and $M$ jump together.

The survival times of a two-dimensional Poisson process actually enjoy a somewhat stronger property than bivariate exponential. Define the piecewise linear function $\Theta: \mathbb{R}_{+} \times \mathbb{R}_{+} \times \mathbb{R} \rightarrow$ $\mathbb{R}_{+}$by

$$
\Theta(s, t, \eta)=\operatorname{Leb}([0, t] \cup[\eta, s+\eta]),
$$

where $L e b$ denotes Lebesgue measure. Notice that $\Theta(s, t, 0)=\max (s, t)$.

Proposition 3.8 (Two-parameter family of survival times). If $(N, M)$ is a two-dimensional Poisson process with survival times $\left(\tau_{u}, \gamma_{v}\right)$, then there are non-negative constants $\lambda, \mu, \rho$ with $\rho \leq \min (\lambda, \mu)$, such that for all $u, v, s, t \geq 0$,

$$
P\left(\tau_{u}>s, \gamma_{v}>t\right)=\exp (-(\lambda-\rho) s-(\mu-\rho) t-\rho \Theta(s, t, u-v)) .
$$

In particular, each member of the diagonal subfamily $\left\{\left(\tau_{u}, \gamma_{u}\right), u \geq 0\right\}$ is bivariate exponential $b \mathcal{E}(\lambda, \mu, \rho)$ in the ordinary sense.

\subsection{Two dimensional Lévy processes}

Theorem 3.7 extends to general Lévy processes, for which we need to extend the notion of joint survival times. For general Lévy processes, these are now dependent on the size of the jumps in each component. For a one dimensional Lévy process, we first summarize some convenient notation and terminology.

First, for $x \in \mathbb{R} \backslash\{0\}$, the tail process $N^{x}$ of $N$ at level $x$ is the counting process defined by

$$
N_{t}^{x}(\omega)=\#\left\{0 \leq s \leq t: \triangle N_{s}(\omega) \in \mathcal{I}(x)\right\}, \quad \omega \in \Omega, t \geq 0
$$


Writing $\mathcal{I}(x)=[x, \infty)$ if $x>0$ and $\mathcal{I}(x)=(-\infty, x]$, if $x<0$, we say that the tail integral induced by $N$ is

$$
\Lambda(x)=\int_{\mathcal{I}(x)} \lambda(d w),
$$

and for $u \geq 0$, the survival time $\tau_{u}^{x}$ of $N$ at level $x$ is

$$
\tau_{u}^{x}=\inf \left\{t \geq 0: N_{t+u}^{x}>N_{u}^{x}\right\} .
$$

If $X$ denotes the TPRM of $N$, and using the notation

$$
X_{t}(\mathcal{I}(x))=X([0, t] \times \mathcal{I}(x)),
$$

we see that the tail process $N^{x}$ of $N$ is nothing more than the Poisson process $\left(X_{t}(\mathcal{I}(x))\right)_{t \geq 0}$ with mean $\Lambda(x)$ and the survival time $\tau_{u}^{x}$ is an exponential random variable with parameter $\Lambda(x)$.

Following our study of two dimensional Poisson processes, we next define the notion of memoryless property for processes.

Definition 3.9 (Two dimensional memoryless property for processes). Let $N$ and $M$ be two one-dimensional Lévy processes. We say that the joint survival times of $N$ and $M$ satisfy the two dimensional memoryless property if: for all $x, y \in \mathbb{R} \backslash\{0\}$, all $u \geq 0$, and all $s_{1}, s_{2}, t \geq 0$,

$$
\mathbb{P}\left(\tau_{u}^{x}>s_{1}+t, \gamma_{u}^{y}>s_{2}+t \mid \tau_{u}^{x}>t, \gamma_{u}^{y}>t\right)=\mathbb{P}\left(\tau_{0}^{x}>s_{1}, \tau_{0}^{y}>s_{2}\right) .
$$

It is straightforward to verify, using Theorem 3.4, that the joint survival times of $N$ and $M$ satisfy the two dimensional memoryless property if and only if for all $x, y \in \mathbb{R} \backslash\{0\}$, there exists $\rho(x, y) \in[0, \min \{\Lambda(x), \Gamma(y)\}]$ such that all the pairs of the collection $\left\{\left(\tau_{u}^{x}, \gamma_{u}^{y}\right), u \geq 0\right\}$ are bivariate exponential with parameters $\Lambda(x), \Gamma(y), \rho(x, y)$.

In case the condition in Definition 3.9 appears to be rather strong, we note that every two-dimensional Lévy process satisfies it.

Proposition 3.10. Let $\left(N_{t}, M_{t}\right)_{t \geq 0}$ be a two dimensional Lévy process. Then the joint survival times of $N$ and $M$ satisfy the memoryless property 3.9.

Proof: Since, for $x, y \in(\mathbb{R} \backslash\{0\})^{2}$, the process $\left(N^{x}, M^{y}\right)$ is a Lévy process with Poisson marginals, we may apply Theorem 3.7.

\subsection{Lévy Copulas}

In this subsection we describe the jump dependence of Lévy processes in terms of Lévy copulas, introduced by Tankov in [25] for Lévy processes with positive jumps and extended to general Lévy processes in [13]. Just as a classical copula connects the distribution of a multivariate random variable to its marginal distributions, in a similar way a Lévy copula connects marginal tail integrals to a multidimensional tail integral.

Definition 3.11 (Tail integral induced by a Lévy measure). Let $\pi$ be a two dimensional Lévy measure. The tail integral $\Pi$ induced by $\pi$ is the function $\Pi:(\mathbb{R} \backslash\{0\})^{2} \rightarrow(0, \infty)$ such that

$$
\Pi(x, y)=\pi(\mathcal{I}(x) \times \mathcal{I}(y)) .
$$

July 2, 2016 
Definition 3.12 ([8, 13], Two dimensional Levy copula). A function $\rho:[-\infty, \infty]^{2} \rightarrow$ $[-\infty, \infty]$ is a Lévy copula if

(i) $\rho(u, v) \neq \infty$ for $(u, v) \neq(\infty, \infty)$

(ii) $\rho(u, v)=0$ if $u=0$ or $v=0$.

(iii) $\rho$ is 2-increasing: if $a_{1} \leq b_{1}$ and $a_{2} \leq b_{2}$, then

$$
\rho\left(a_{1}, a_{2}\right)+\rho\left(b_{1}, b_{2}\right)-\rho\left(a_{1}, b_{2}\right)-\rho\left(b_{1}, a_{2}\right) \geq 0 .
$$

(iv) $\rho(\infty, u)-\rho(-\infty, u)=\rho(u, \infty)-\rho(u,-\infty)=u, u \in[0, \infty)$.

Example 3.13 (Archimedian Lévy copulae). Examples of two dimensional Lévy copulae can be constructed [25] from a generator function $\phi$ with

$$
\rho\left(u_{1}, u_{2}\right)=\phi^{-1}\left(\phi\left(u_{1}\right)+\phi\left(u_{2}\right)\right)\left(1_{\{u v \geq 0\}}-1_{\{u v<0\}}\right)
$$

so that $\rho$ satisfies Def. 3.12.

(i) The Clayton Lévy copula

$$
\rho(u, v)=\left(|u|^{-\theta}+|v|^{-\theta}\right)^{-1 / \theta}\left(1_{\{u v \geq 0\}}-1_{\{u v<0\}}\right),
$$

for values of the parameters $\theta>0$ was introduced in [25]. It has generator $\phi(\theta)=|u|^{-\theta}$.

(ii) The Frank Lévy copula with generator $\phi(u)=-\log \left(1-e^{-\eta|u|}\right), \eta>0$, is

$$
\rho(u, v)=-\frac{1}{\eta} \log \left\{1-\left(1-e^{-\eta|u|}\right)\left(1-e^{-\eta|v|}\right)\right\}\left(1_{\{u v \geq 0\}}-1_{\{u v<0\}}\right) .
$$

In our setting we will only be interested in $u, v<0$ (corresponding to negative jumps), so in that case the term $\left(1_{\{u v \geq 0\}}-1_{\{u v<0\}}\right)=1$ and can be ignored in the examples above. We can restrict attention to Lévy copulas $\rho:[-\infty, 0]^{2} \rightarrow[0, \infty]$.

Notation: Let sgn be the function that returns the sign of a real number, i.e. $\operatorname{sgn}(x)=1$ if $x>0$ and $\operatorname{sgn}(x)=-1$ if $x<0$. We denote by $\bar{\Pi}$ the function such that, for $(x, y) \in(\mathbb{R} \backslash\{0\})^{2}$, $\bar{\Pi}(x, y)=\operatorname{sgn}(x y) \Pi(x, y)$. Similarly, if $\Lambda$ is a one dimensional tail integral, we denote $\bar{\Lambda}$ the function such that, $x \in \mathbb{R} \backslash\{0\}, \bar{\Lambda}(x)=\operatorname{sgn}(x) \Lambda(x)$.

The fundamental theorem for Lévy copulas is the following.

Theorem 3.14 ([13], Sklar-Kallsen-Tankov theorem for Lévy processes). Let $Y=(N, M)$ be an $\mathbb{R}^{2}$-valued Lévy process with Lévy measure $\pi$. Let $\Pi$ be the tail integral induced by $\pi$ with marginal tail integrals $\Lambda$ and $\Gamma$. Then, there exists a Lévy copula $\rho$ so that for all $x, y \in(\mathbb{R} \backslash\{0\})^{2}$,

$$
\bar{\Pi}(x, y)=\rho(\bar{\Lambda}(x), \bar{\Gamma}(y)) .
$$

If $\Lambda$ and $\Gamma$ are continuous, then $\rho$ is unique. Otherwise, it is unique on Range $(\Lambda) \times \operatorname{Range}(\Gamma)$.

Conversely, Let $\rho$ be a 2-dimensional Lévy copula and let $N$ and $M$ be two one dimensional Lévy processes with respective tail integrals $\Lambda$ and $\Gamma$. Then there exists a two dimensional Lévy process $Y$ with marginal tail integrals $\Lambda$ and $\Gamma$ and Lévy copula $\rho$. The tail integral $\Pi$ induced by the Lévy measure of $Y$ is given by (33).

July 2, 2016 
Theorem 3.14 states that a two-dimensional Lévy measure can be constructed starting from either sides of Eq. (33). Given two one dimensional Lévy measures $\lambda$ and $\mu$, there exists a two dimensional Lévy measure $\pi$ such that the margins of $\pi$ are precisely $\lambda$ and $\mu$, and $\forall(x, y) \in(\mathbb{R} \backslash\{0\})^{2}$,

$$
\pi(\mathcal{I}(x) \times \mathcal{I}(y))=\Pi(x, y)=\operatorname{sgn}(x y) \rho(\bar{\Lambda}(x), \bar{\Gamma}(y)) .
$$

The meaning of (34) is that the intensity of the jumps of a two dimensional Lévy process $Y=(N, M)$ jointly greater than $(x, y)$ is a function of the intensities of the jumps in $N$ and $M$ larger than $x$ and $y$ respectively. For our application to credit risk, we pay particular attention to pairs $(x, y)$ in the third quadrant, i.e. $(x, y) \in(-\infty, 0)^{2}$. In this case, Eq. (34) becomes

$$
\Pi(x, y)=\pi((-\infty, x] \times(-\infty, y])=\rho(\bar{\Lambda}(x), \bar{\Gamma}(y)) .
$$

\subsection{Dependence Theorem}

The following Theorem summarizes the main results of this section.

Theorem 3.15 (Dependent Lévy processes and survival times). Let $N$ and $M$ be two one dimensional Lévy processes on $\left(\Omega, \mathcal{F}, \mathbb{P},\left(\mathcal{F}_{t}\right)_{t \geq 0}\right)$ with respective Lévy measures $\lambda$ and $\mu$ and tail integrals $\Lambda$ and $\Gamma$.

Suppose $Y=(N, M)$ is a two dimensional Lévy process. Let $X$ denote its TPRM, with intensity $\pi$, and tail integral

$$
\Pi(x, y)=\mathbb{E} X_{1}(\mathcal{I}(x) \times \mathcal{I}(y))=\pi(\mathcal{I}(x) \times \mathcal{I}(y)), \quad x, y \in \mathbb{R} \backslash\{0\} .
$$

Then the following statements hold.

(i) - The joint survival times of $N$ and $M$ satisfy the two-dimensional memoryless property.

(ii) - for each $x, y \in \mathbb{R} \backslash\{0\}$, all the pairs of the collection $\left\{\left(\tau_{u}^{x}, \gamma_{u}^{y}\right), u \geq 0\right\}$ are bivariate exponential with parameters $\Lambda(x), \Gamma(y)$ and $\Pi(x, y)$.

(iii) - For $u \geq 0$, the joint survival times $\left(\tau_{u}^{x}, \gamma_{u}^{y}\right)$ of $N$ and $M$ at the level $(x, y)$ are such that,

$$
\forall s, t \in \mathbb{R}_{+}, \quad \mathbb{P}\left(\tau_{u}^{x}>s, \gamma_{u}^{y}>t\right)=C_{\rho}^{\lambda, \mu}\left(e^{-\Lambda(x) s}, e^{-\Gamma(y) t}\right),
$$

where $C_{\rho}^{\lambda, \mu}$ is the 3 parameter Marshall-Olkin copula 3.3 with parameters

$$
\rho=\Pi(x, y), \quad \lambda=\Lambda(x), \quad \text { and } \quad \mu=\Gamma(y) .
$$

(iv) - There exists a Lévy copula $L:[-\infty, \infty]^{2} \rightarrow[-\infty, \infty]$ such that

$$
\forall x, y \in \mathbb{R} \backslash\{0\}, \quad \Pi(x, y)=\operatorname{sgn}(x y) L(\bar{\Lambda}(x), \bar{\Gamma}(y)) .
$$

A few things are worth pointing out here. First, this Theorem applies generally to all two-dimensional Lévy processes $Y=(N, M)$. Despite that generality, the joint survival probabilities have a very specific structure, in which the marginal survival probabilities are always coupled by a Marshall-Olkin copula with parameters determined by the Lévy measure $\pi$ of $Y$ and the Lévy measures of the margins $N$ and $M$. The three Marshall-Olkin parameters are simply given by the three tail integrals of $Y, N$, and $M$, and so the dependence of $\rho(x, y), \lambda(x), \mu(y)$ on $x$ and $y$ is completely determined by $\pi$. 
From the modeling perspective, this conveniently provides an explicit expression for the joint survival probabilities, and the modeler can choose or fit the marginal processes separately from the choice of Lévy copula describing the jump dependence. This is directly analogous to building a multivariate probability distribution by choosing the margins and the (classical) copula separately.

\section{A Multi-name structural default model}

In order to price basket credit derivatives, we need the two dimensional version of our default survival probability formulas, presented here. We work on the complete filtered probability space $\left(\Omega, \mathcal{F}, \mathbb{P},\left(\mathcal{F}_{t}\right)_{t \geq 0}\right)$ and assume that the two stock prices $S^{1}$ and $S^{2}$ of firm 1 and firm 2, respectively, can be written in terms of a two dimensional Lévy process $Y=\left(Y^{1}, Y^{2}\right)$

$$
\forall \omega \in \Omega, t \in \mathbb{R}_{+}, \quad S_{t}^{1}(\omega)=e^{r t+Y_{t}^{1}(\omega)+t \psi^{1}(-\mathbf{i})}, \quad S_{t}^{2}(\omega)=e^{r t+Y_{t}^{2}(\omega)+t \psi^{2}(-\mathbf{i})},
$$

where $\psi^{i}$ is the characteristic exponent of $Y_{1}^{i}, i=1,2$, and $\mathbf{i}$ is the imaginary unit.

We denote by $X$ the TPRM on $E=\mathbb{R}^{2} \backslash\{0\}$ of $Y$, and by $\pi$ the Lévy measure of $Y$, satisfying $\pi\left[|x|^{2} \wedge 1\right]<\infty$.

The tail integral 3.11 of $Y$ is the function $\Pi:(\mathbb{R} \backslash\{0\})^{2} \rightarrow(0, \infty)$ such that

$$
\forall(x, y) \in E, \quad \Pi(x, y)=\pi(\mathcal{I}(x) \times \mathcal{I}(y))=\int_{\mathcal{I}(x) \times \mathcal{I}(y)} \pi(d u \times d v),
$$

and we write $\bar{\Pi}(x, y)=\operatorname{sgn}(x y) \Pi(x, y)$.

Likewise, for the one dimensional Lévy processes $Y^{1}$ and $Y^{2}$, we denote the respective Lévy measures $\lambda$ and $\mu$, and the tail integrals by $\Lambda$ and $\Gamma$, with $\bar{\Lambda}(x)=\operatorname{sgn}(x) \Lambda(x)$ and $\bar{\Gamma}(x)=\operatorname{sgn}(x) \Gamma(x)$.

As in Definition 2.1, the default of firm $i \in\{1,2\}$ is the first time the log-return of its stock price jumps to or below the default level $a_{t}^{i}$, with default times

$$
\tau_{i}(\omega)=\inf \left\{t>0: \log \left(S_{t}^{i}(\omega) / S_{t^{-}}^{i}(\omega)\right) \leq a_{t}^{i}(\omega)\right\}, \quad i=1,2 .
$$

Proposition 4.1 (Joint survival probability, stochastic level). For $i=1,2$, let $a_{t}^{i}$ be a default level and $\tau_{i}$ the default time for $S^{i}$, as in (41), where the stock prices $S^{i}$ are given by (39) and driven by a two-dimensional Lévy process $Y$ with tail integral $\Pi$ and marginal tail integrals $\Lambda$ and $\Gamma$. Assume the default levels are both independent of $Y$.

Then there exists a Lévy copula $\rho(\cdot, \cdot)$ such that $\rho(\bar{\Lambda}(x), \bar{\Gamma}(y))=\Pi(x, y)$ and $\forall s, t \geq 0$,

$$
\begin{gathered}
\mathbb{P}\left(\tau_{1}>s, \tau_{2}>t\right)=\mathbb{E}\left[\exp -\left\{\int_{0}^{s} \Lambda\left(a_{u}^{1}\right) d u+\int_{0}^{t} \Gamma\left(a_{u}^{2}\right) d u-\int_{0}^{s \wedge t} \Pi\left(a_{u}^{1}, a_{u}^{2}\right) d u\right\}\right] \\
=\mathbb{E}\left[\exp -\left\{\int_{0}^{s} \Lambda\left(a_{u}^{1}\right) d u+\int_{0}^{t} \Gamma\left(a_{u}^{2}\right) d u-\int_{0}^{s \wedge t} \rho\left(\bar{\Lambda}\left(a_{u}^{1}\right), \bar{\Gamma}\left(a_{u}^{2}\right)\right) d u\right\}\right]
\end{gathered}
$$

The proof (see Section 7) proceeds by establishing the case of deterministic default level first. 
Corollary 4.2 (Joint survival probablity, deterministic default level). Let $\tau_{1}$ and $\tau_{2}$ be the default times, for deterministic default levels $a^{1}, a^{2}$, of two firms with respective stock prices given by (39) driven by a two-dimensional Lévy process $Y$ with marginal tail integrals $\Lambda$ and $\Gamma$. Then there exists a Lévy copula $\rho(\cdot, \cdot)$ such that $\rho(\bar{\Lambda}(x), \bar{\Gamma}(y))=\Pi(x, y)$ and $\forall s, t \geq 0$,

$$
\mathbb{P}\left(\tau_{1}>s, \tau_{2}>t\right)=\exp -\left\{\int_{0}^{s} \Lambda\left(a_{u}^{1}\right) d u+\int_{0}^{t} \Gamma\left(a_{u}^{2}\right) d u-\int_{0}^{s \wedge t} \rho\left(\bar{\Lambda}\left(a_{u}^{1}\right), \bar{\Gamma}\left(a_{u}^{2}\right)\right) d u\right\} .
$$

Recall the notations $x \wedge y=\min (x, y)$ and $x \vee y=\max (x, y)$. The previous corollary gives information about the distribution of the first default time $\tau^{(1)}=\tau_{1} \wedge \tau_{2}$. Since $\mathbb{P}\left(\tau^{(1)}>t\right)=$ $\mathbb{P}\left(\tau_{1}>t, \tau_{2}>t\right)$, we immediately obtain

Corollary 4.3 (First-to-default survival probability, deterministic default level). Let $\tau_{1}$ and $\tau_{2}$ be the default times, for deterministic default levels $a_{t}^{1}, a_{t}^{2}$, of two firms with respective stock prices given by (39) driven by a two-dimensional Lévy process $Y$ with marginal tail integrals $\Lambda$ and $\Gamma$. Then there exists a Lévy copula $\rho(\cdot, \cdot)$ such that $\rho(\bar{\Lambda}(x), \bar{\Gamma}(y))=\Pi(x, y)$ and

$$
\forall t \geq 0, \quad \mathbb{P}\left(\tau^{(1)}>t\right)=\exp -\int_{0}^{t} \Lambda\left(a_{s}^{1}\right)+\Gamma\left(a_{s}^{2}\right)-\rho\left(\bar{\Lambda}\left(a_{s}^{1}\right), \bar{\Gamma}\left(a_{s}^{2}\right)\right) d s .
$$

These formulas become simplified in case the default levels $a^{1}, a^{2}$ are constant:

$$
\begin{aligned}
\mathbb{P}\left(\tau_{1}>s, \tau_{2}>t\right) & =C_{\rho}^{\lambda, \mu}\left(e^{-\Lambda\left(a_{1}\right) s}, e^{-\Gamma\left(a_{2}\right) t}\right) \\
& =\exp -\left\{s \Lambda\left(a_{s}^{1}\right)+t \Gamma\left(a_{t}^{2}\right)-(s \wedge t) \rho\left(\bar{\Lambda}\left(a_{(s \wedge t)}^{1}\right), \bar{\Gamma}\left(a_{(s \wedge t)}^{2}\right)\right)\right\} .
\end{aligned}
$$

where $C_{\rho}^{\lambda, \mu}$ is the 3 parameter Marshall-Olkin copula of Definition 3.3 with

$$
\lambda=\Lambda\left(a_{1}\right), \quad \mu=\Gamma\left(a_{2}\right), \quad \text { and } \quad \rho=\rho\left(\bar{\Lambda}\left(a_{1}\right), \bar{\Gamma}\left(a_{2}\right)\right) .
$$

Equivalently, $\left(\tau_{1}, \tau_{2}\right)$ is bivariate exponential $b \mathcal{E}(\lambda, \mu, \rho)$. Likewise the first-to-default distribution simplifies to

$$
\mathbb{P}\left(\tau^{(1)} \leq t\right)=1-\exp -t\left\{\Lambda\left(a_{1}\right)+\Gamma\left(a_{2}\right)-\rho\left(\bar{\Lambda}\left(a_{1}\right), \bar{\Gamma}\left(a_{2}\right)\right)\right\} .
$$

To gain some intuition, we can compute the joint hazard rate, stated here in the simplest case.

Proposition 4.4 (Joint hazard rate, constant default levels). For constant default levels $a_{1}, a_{2}$, the joint hazard rate $J H_{t}$ is given by

$$
J H_{t} \equiv \lim _{h \downarrow 0} \frac{\mathbb{P}\left(\tau_{1} \vee \tau_{2} \leq t+h \mid \tau_{1} \wedge \tau_{2}>t\right)}{h}=\rho\left(\bar{\Lambda}\left(a_{1}\right), \bar{\Gamma}\left(a_{2}\right)\right)
$$

or, equivalently,

$$
J H_{t}=\pi\left(\left(-\infty, a_{1}\right) \times\left(-\infty, a_{2}\right)\right) .
$$

Proposition 4.4 means that the Lévy copula $\rho$ is responsible for the instantaneous joint default of both firms. For example, in the case of the independent Lévy copula $\rho^{\perp}$,

$$
\forall(u, v) \in[-\infty, \infty]^{2}, \quad \rho^{\perp}(u, v)=u 1_{[v=\infty]}+v 1_{[u=\infty]},
$$

the joint local default rate is 0 , and the default times $\tau_{1}$ and $\tau_{2}$ are independent. 
Example 4.5. Consider the commonly used case of piecewise constant default levels. Let $0=t_{0}<t_{1}<\ldots<t_{I}$ and define the default levels $a_{t}^{i}, i \in\{1,2\}$ such that

$$
\forall t \geq 0, \quad a_{t}^{i}=\sum_{k=1}^{K-1} a_{k}^{i} 1_{\left[t_{i-1}, t_{i}\right)}(t)+a_{K}^{i} 1_{t \geq t_{I}}
$$

where $a_{k}<0, i=1, \ldots, K$. For $t \in\left[t_{k}, t_{k+1}\right), k=1, \ldots, K-1$, the survival probability of the FtD up to time $t$ is then

$$
\mathbb{P}\left(\tau^{(1)}>t\right)=\exp \left\{-\sum_{i=1}^{k} \theta_{i}\left(t_{i}-t_{i-1}\right)-\theta_{k}\left(t-t_{k}\right)\right\},
$$

where the constants $\theta_{k}$ are given by

$$
\theta_{k}=\Lambda_{1}\left(a_{k}^{1}\right)+\Lambda_{2}\left(a_{k}^{2}\right)-\rho\left(\bar{\Lambda}_{1}\left(a_{k}^{1}\right), \bar{\Lambda}_{2}\left(a_{k}^{2}\right)\right) .
$$

\section{Pricing of a first to default basket CDS}

In this section, we illustrate the pricing of a First-to-Default swap (FtD) with the multidimensional structural jump threshold framework we have developed. We discuss how the modeler can choose a particular Lévy copula to obtain a spectrum of dependence regimes, separately from the choice of the individual stock price processes. We also give some examples of the first-to-default spread term structures.

\subsection{The model set-up}

A First-to-Default (FtD) swap is a contract which protects against the first default observed in a basket of defaultable bonds. A spread over the risk free rate is paid periodically on the notional value of the contract. When default occurs, the difference between the recovery value of the bond and its face value is paid back to the holder of the FtD.

We assume there are two defaultable bonds in the CDS basket. Each bond has the same recovery value $R$. We denote by $\tau_{1}$ and $\tau_{2}$ the default times of firm 1 and 2 , respectively, and $\tau^{(1)}$ the time of the first default in the basket, $\tau^{(1)}=\min \left(\tau_{1}, \tau_{2}\right)$. The two legs of an FtD are shown in Figure 3.

In order to price this 2-defaultable bond FtD, we assume that the default free rate $r>0$ is constant, and that the protection buyer pays the spread $m^{(1)}$ continuously until the maturity $T$ of the contract. The price of the FtD contract is such that both legs have the same present value (see Fig. 3). If we denote $\mathbb{P}$ the risk neutral probability, the spread of an FtD is such that the value of the default leg $D L_{t}$ at time $t$ is equal to the fixed leg $F L_{t}$. Explicitly,

$$
\begin{aligned}
& D L_{t}=(1-R) \int_{0}^{t} e^{-r s} d \mathbb{P}\left(\tau^{(1)} \leq s\right) \\
& F L_{t}=m^{(1)} \int_{0}^{t} e^{-r s} \mathbb{P}\left(\tau^{(1)}>s\right) d s .
\end{aligned}
$$

Integration by parts for the expression of the default leg gives:

$$
D L_{t}=(1-R)\left[\left(e^{-r t}\left(1-\mathbb{P}\left(\tau^{(1)}>t\right)\right)-\left(e^{-r t}-1\right)-r \int_{0}^{t} e^{-r s} \mathbb{P}\left(\tau^{(1)}>s\right) d s\right]\right.
$$

July 2, 2016 


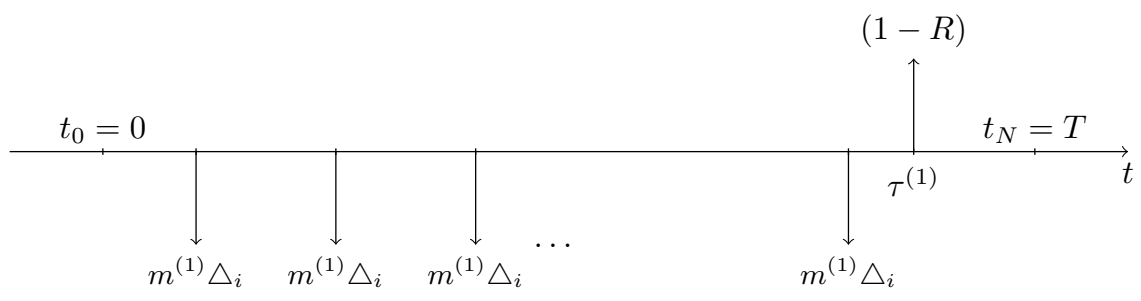

Figure 3: Cash flows involved in a First-to-Default swap. Above $T$ is the maturity of the contract, $R$ is the recovery rate, $\triangle_{i}=t_{i}-t_{i-1}$.

so that the FtD spread at par is

$$
m^{(1)}=(1-R) \frac{1-e^{-r T} \mathbb{P}\left(\tau^{(1)}>T\right)-r \int_{0}^{T} e^{-r s} \mathbb{P}\left(\tau^{(1)}>s\right) d s}{\int_{0}^{T} e^{-r s} \mathbb{P}\left(\tau^{(1)}>s\right) d s} .
$$

In order to value this contract, one therefore only needs to know the joint survival probability $\mathbb{P}\left(\tau_{1}>s, \tau_{2}>t\right)$. We have already solved this problem explicitly in the previous section.

In summary, we model the dynamics of the stock prices $S_{t}^{1}, S_{t}^{2}$ of firm 1 and 2 as exponential Lévy according to

$$
\forall \omega \in \Omega, t \in \mathbb{R}_{+}, \quad S_{t}^{j}(\omega)=e^{r t+Y_{t}^{j}(\omega)+t \psi^{j}(-i)}, \quad j=1,2
$$

where the process $Y=\left(Y^{1}, Y^{2}\right)$ is a two dimensional Lévy process. We denote by $\lambda$ the (two dimensional) Lévy measure of $Y$, which is determined uniquely by the Lévy measures $\lambda_{1}, \lambda_{2}$ of $Y^{1}$ and $Y^{2}$, respectively, along with a Lévy copula $\rho$ describing the dependence between the jumps of $Y^{1}$ and $Y^{2}$ (hence $S^{1}$ and $S^{2}$ ).

In our structural model, the default times are the first time the stock returns cross the default levels $a_{1}$ and $a_{2}$ respectively:

$$
\tau_{i}=\inf \left\{t>0: \log \left(S_{t}^{i} / S_{t^{-}}^{i}\right) \leq a_{i}\right\}, \quad i=1,2 .
$$

With this set-up, Corollary 2.3 gives the survival probability of each firm up to time $0<t<T$,

$$
\mathbb{P}\left(\tau_{i}>t\right)=e^{-t \Lambda_{i}\left(a_{i}\right)},
$$

where $\Lambda_{i}=\int_{-\infty}^{a_{i}} \lambda_{i}(d w)$ is the tail integral of the process $Y^{i}$.

Furthermore Corollary 4.3 gives us the first-to-default probabilities

$$
\mathbb{P}\left(\tau^{(1)}>t\right)=\exp -t\left\{\Lambda_{1}\left(a_{1}\right)+\Lambda_{2}\left(a_{2}\right)-\rho\left(\bar{\Lambda}_{1}\left(a_{1}\right), \bar{\Lambda}_{2}\left(a_{2}\right)\right)\right\},
$$

where recall that $\bar{\Lambda}_{i}\left(a_{i}\right)=-\Lambda_{i}\left(a_{i}\right)$ since $a_{i}<0$. 
This leads us to a simple explicit expression for the FtD spread:

$$
m^{(1)}=(1-R)\left[\Lambda_{1}\left(a_{1}\right)+\Lambda_{2}\left(a_{2}\right)-\rho\left(\bar{\Lambda}_{1}\left(a_{1}\right), \bar{\Lambda}_{2}\left(a_{2}\right)\right)\right] .
$$

To see this, we use equation (56) to determine $m^{(1)}$, where the survival probability is given by equation (60). For notational convenience, define

$$
\Theta=\Lambda_{1}\left(a_{1}\right)+\Lambda_{2}\left(a_{2}\right)-\rho\left(\bar{\Lambda}_{1}\left(a_{1}\right), \bar{\Lambda}_{2}\left(a_{2}\right)\right) .
$$

From the equation(60), we have

$$
\mathbb{P}\left(\tau^{(1)}>t\right)=e^{-t \Theta},
$$

so we could call $\Theta$ the "intensity" of the first-to-default process, even though we have a structural default framework. We have

$$
m^{(1)}=(1-R) \frac{\left[1-e^{-r T} e^{-\Theta T}-r \int_{0}^{T} e^{-r s} e^{-\Theta s} d s\right]}{\int_{0}^{T} e^{-r s} e^{-\Theta s} d s} .
$$

These integrals are easily computed and the expression simplifies to

$$
m^{(1)}=(1-R) \Theta,
$$

which is equation (61).

We can describe, via equation (61), two limiting cases of dependence. Since Lévy copulas are bounded from above and from below [13], one can explicitly write an expression for $m^{(1)}$ when $S^{1}$ and $S^{2}$ are

(I) Independent. The Lévy copula $\rho$ is at its lower bound:

$$
\rho(u, v)=u 1_{[v=\infty]}+v 1_{[u=\infty]} .
$$

Then,

$$
m^{(1)}=(1-R)\left\{\Lambda_{1}\left(a_{1}\right)+\Lambda_{2}\left(a_{2}\right)-\left(\Lambda_{1}\left(a_{1}\right) 1_{\left[\Lambda_{2}\left(a_{2}\right)=\infty\right]}+\Lambda_{2}\left(a_{2}\right) 1_{\left[\Lambda_{1}\left(a_{1}\right)=\infty\right]}\right)\right\},
$$

and since $a_{1}, a_{2}<0$, this gives us

$$
m^{(1)}=(1-R)\left\{\Lambda_{1}\left(a_{1}\right)+\Lambda_{2}\left(a_{2}\right)\right\}=m_{1}+m_{2} .
$$

In the equation above, $m_{1}$ and $m_{2}$ are the par spreads of CDS contracts on firm 1 and firm 2 respectively. We therefore obtain that the price of the protection against the first-to-default two-defaultable basket, when the two underlyings are independent, is equal to the sum of the protections against each, respectively.

(II) Dependent. The Lévy copula $\rho$ is at its upper bound

$$
\rho(u, v)=\min (u, v) .
$$

This yields the following expression for $m^{(1)}$,

$$
m^{(1)}=(1-R) \max \left(\Lambda_{1}\left(a_{1}\right), \Lambda_{2}\left(a_{2}\right)\right)=\max \left(m_{1}, m_{2}\right) .
$$

Therefore when the two underlyings are completely dependent, the price of the protection against the first to default in the basket is equal to the most expensive of the two premia on each of the underlyings. 


\subsection{Choosing a dependence structure between stock prices}

To illustrate the complete computation of the FtD CDS spread, we now make some explicit choices for the model inputs. We assume that $\lambda_{1}$ and $\lambda_{2}$ are the Lévy measures of two one dimensional $\alpha$-stable Lévy processes:

$$
\lambda_{i}(d x)=\alpha_{i}\left(\frac{c_{+}^{i}}{x^{1+\alpha_{i}}} 1_{x>0}+\frac{c_{-}^{i}}{|x|^{1+\alpha_{i}}} 1_{x<0}\right) d x
$$

with $1<\alpha_{i}<2$ and $c_{+}^{i}, c_{-}^{i} \geq 0$. The fact that $\alpha_{i} \in(1,2)$ places $S^{1}$ and $S^{2}$ in a regime of infinite activity, i.e. that almost surely $S^{1}$ and $S^{2}$ have infinitely many jumps in compact time intervals.

Since the measures $\lambda_{1}$ and $\lambda_{2}$ model the stock prices, these should be calibrated separately either on historical log returns or option prices [8].

The tail integrals $\Lambda_{1}$ and $\Lambda_{2}$ of $Y^{1}$ and $Y^{2}$ respectively are of the form

$$
\forall x<0, \quad \Lambda_{i}(x)=\int_{-\infty}^{x} \lambda_{i}(d u)=\frac{c_{-}^{i}}{|x|^{\alpha_{i}}} .
$$

To model the jump dependence, we choose the Frank Lévy copula

$$
\rho(u, v)=-\frac{1}{\eta} \log \left\{1-\left(1-e^{-\eta|u|}\right)\left(1-e^{-\eta|v|}\right)\right\} .
$$

The Frank Lévy copula is symmetric and parametrized by $\eta>0$.

We first illustrate the set-up of this framework in the case where the default levels $a_{1}, a_{2}$ are constant. In this case, we have seven parameters: our individual stock parameters $\alpha_{i}, c_{-}^{i}$, $i=1,2$, the jump dependence parameter $\eta$, and the constant default levels $a_{1}, a_{2}$. We might expect the default levels to be estimated from single-name CDS prices, and the dependence parameter from, for example, existing market basket CDS prices.

The FtD par spread now comes from equation (61):

$$
m^{(1)}=(1-R) \Theta
$$

where the default intensity $\Theta$ is given by

$$
\Theta=\Lambda_{1}\left(a_{1}\right)+\Lambda_{2}\left(a_{2}\right)-(1 / \eta) \log \left[1-\left(1-e^{-\eta \Lambda_{1}\left(a_{1}\right)}\right)\left(1-e^{-\eta \Lambda_{2}\left(a_{2}\right)}\right)\right],
$$

and

$$
\Lambda_{i}\left(a_{i}\right)=\frac{c_{-}^{i}}{\left|a_{i}\right|^{\alpha_{i}}}, \quad i=1,2
$$

The joint survival probability of firm 1 and firm 2 until time $T>0$ comes from Equation (46). We show this probability distribution in Figure 4 as a function of the default levels $a_{1}$ and $a_{2}$ for a fixed maturity of $T=1$ year and for various values of the dependence parameter $\eta$. Smaller values of default levels lead to an immediate default while higher values means a lower probability of default. The parameter $\eta$ controls the shape of the joint distribution.

When jumps of $S^{1}$ and $S^{2}$ are nearly independent, $\eta$ is near zero, and the first order approximation of the intensity for small $\eta$ gives us this simple linear formula for the dependence of the spread on the jump dependence parameter:

$$
m^{(1)} \approx(1-R)\left[\Lambda_{1}\left(a_{1}\right)+\Lambda_{2}\left(a_{2}\right)+\eta \Lambda_{1}\left(a_{1}\right) \Lambda_{2}\left(a_{2}\right)\right] .
$$




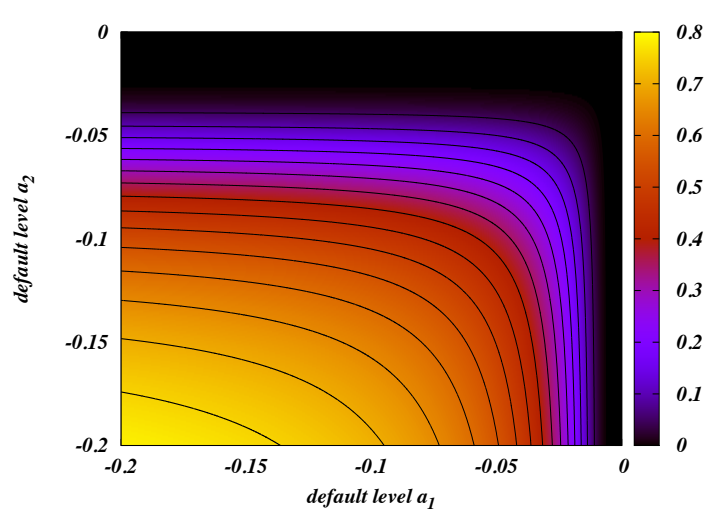

(a) $\eta=0.01$

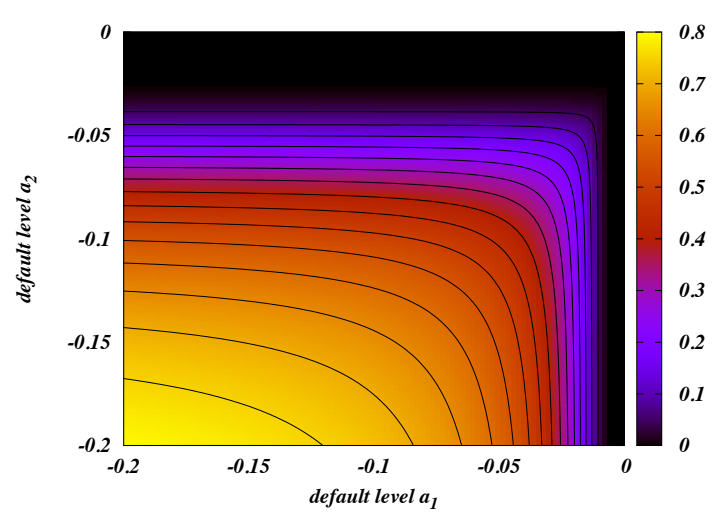

(b) $\eta=1$

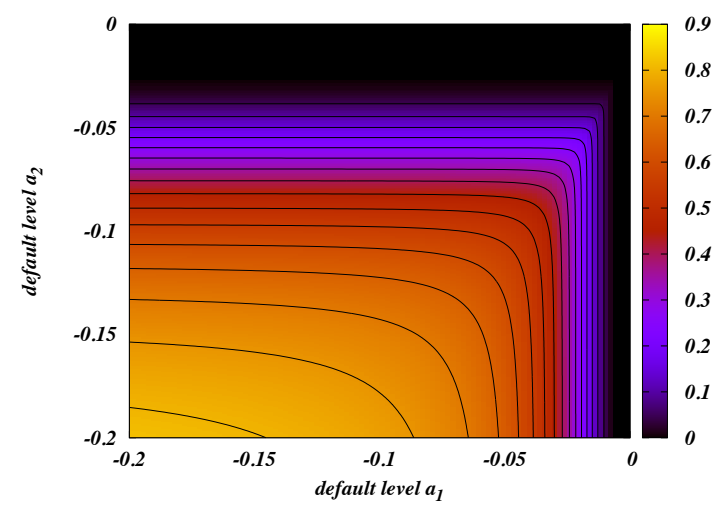

(c) $\eta=5$

Figure 4: Joint default probability from Equation (46) at the level $a_{1}, a_{2}$. The dependence is given by the Frank-Lévy copula Eq. (71). Tail integrals are $\alpha$-stable, given in Eq. (70). We choose $\alpha_{1}=3 / 2, \alpha_{2}=7 / 4, c_{1}^{-}=c_{2}^{-}=1 / 100$. Small values of $\eta$ correspond to the independent case. Larger values means that a simultaneous default is more likely.

We conclude by computing the FtD spreads given in Eq. (56) for different maturities, when the default level $a(t)$ is a piecewise constant deterministic function. We assume that the underlying stock process of each firm is an exponential $\alpha$-stable process, with Lévy measure given by Eq. (69). The tail integral corresponding to jumps in each component is therefore given by Eq. (70). For this example, we choose the parameters $\alpha_{1}=\alpha_{2}=1.25$, and $c_{-}^{1}=c_{-}^{2}=1 / 100$. The default level $a(t)$ is given in Fig. 5(a). The remaining parameter to choose is then the dependence parameter $\eta$. We show Fig. 5(b) the term structure of FtD spreads for three different values of $\eta$. By changing the value of this parameter, one can obtain the entire spectrum of dependence regimes, from independence (see Eq. 66) to the complete dependence case (see Eq. 67).

We note in closing that all these computations are straightforward and fast because we need only implement explicit formulas for spread and survival probabilities in terms of our explicit tail integrals. 


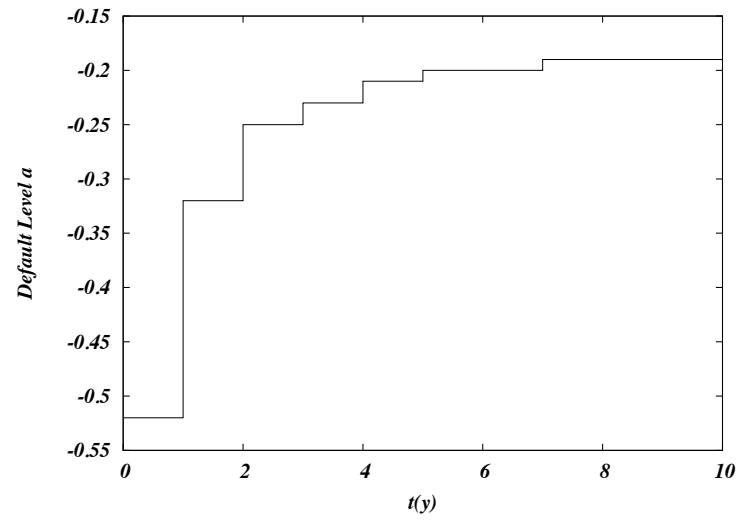

(a) Piecewise constant default level.

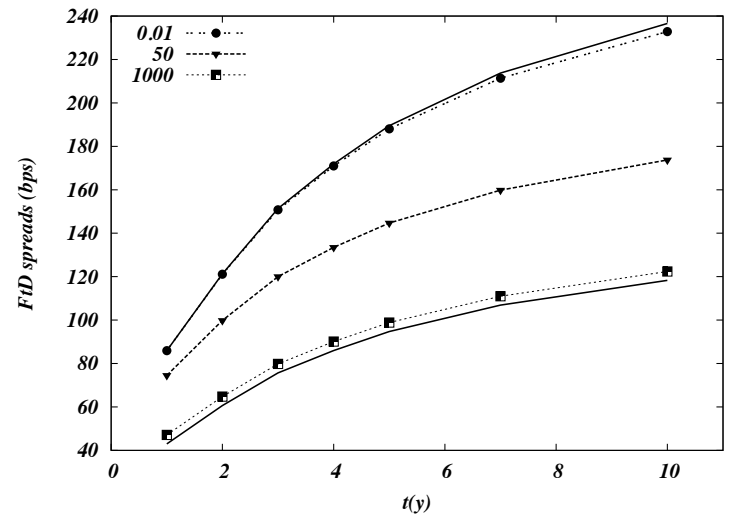

(b) First-to-Default spreads in the case of a piecewise constant default level, for $\eta \in\{0.001,50,1000\}$. The solid curves are the limiting cases $\max \left(m_{t}^{1}, m_{t}^{2}\right)$ and $m_{t}^{1}+m_{t}^{2}$.

Figure 5: First-to-Default spreads $m_{t}^{(1)}$ in Eq. (56) (right) in the set-up of our structural jump threshold framework with piecewise constant default levels (left). The dependence between firms is given by the Frank-Lévy copula Eq. (71). Tail integrals are $\alpha$-stable, given in Eq. (70). We choose $\alpha_{1}=\alpha_{2}=3 / 2, c_{1}^{-}=c_{2}^{-}=1 / 100$. A high value of the parameter $\eta$ leads to a strong dependence and the FtD spread $m_{t}^{(1)}$ tends to $\max \left(m_{t}^{1}, m_{t}^{2}\right)$. When $\eta$ is small, this is the independence case, $m_{t}^{(1)} \rightarrow m_{t}^{1}+m_{t}^{2}$. 


\section{Concluding remarks}

The results above take advantage of recent advances in understanding the jump dependence of Lévy processes to formulate a multi-dimensional structural default model framework that has explicit pricing formulas similar to those of familiar reduced form models. One way to think of this is that it is a way to interpret a reduced form model in terms of the underlying price processes, which provides a way to link in a consistent way the prices of various credit and option models with a common view of the underliers.

The results we have presented raise several additional questions. For the case $N>2$, the theory and formulas work out in the same way, except the notation becomes more burdensome. However, since basket credit derivatives usually involve more than two underliers, a typical application will require working things out for larger $N$. As a hint for how the formulas look, we mention here without proof the formula for the survival probability of the first-to-default time for $N=3$ and constant default levels $a_{1}, a_{2}, a_{3}$.

As before, one needs to calibrate three Lévy measures $\lambda_{1}, \lambda_{2}$ and $\lambda_{3}$, and choose a Lévy copula $L$ in order to construct the full three-dimensional tail integral $\Pi$. The two-dimensional marginal tail integrals $\Pi_{i, j}$, in dimensions $i, j$, are then obtained from $\Pi$, and we can write the survival probability of the first-to-default time as:

$$
\begin{aligned}
\mathbb{P}\left(\tau^{(1)}>t\right)= & \exp \left[-t\left\{\Lambda_{1}\left(a_{1}\right)+\Lambda_{2}\left(a_{2}\right)+\Lambda_{3}\left(a_{3}\right)\right.\right. \\
& \left.\left.-\Pi_{1,2}\left(a_{1}, a_{2}\right)-\Pi_{1,3}\left(a_{1}, a_{3}\right)-\Pi_{2,3}\left(a_{2}, a_{3}\right)+\Pi\left(a_{1}, a_{2}, a_{3}\right)\right\}\right]
\end{aligned}
$$

or

$$
\mathbb{P}\left(\tau^{(1)}>t\right)=\exp \left\{-t\left\{\mathbf{1}^{t} \Lambda^{(1)} \mathbf{1}+\Pi\left(a_{1}, a_{2}, a_{3}\right)\right\}\right\}
$$

where $\Lambda^{(1)}$ is the matrix

$$
\Lambda^{(1)}=\left[\begin{array}{ccc}
\Lambda_{1}\left(a_{1}\right) & -\Pi_{1,2}\left(a_{1}, a_{2}\right) & -\Pi_{1,3}\left(a_{1}, a_{3}\right) \\
0 & \Lambda_{2}\left(a_{2}\right) & -\Pi_{2,3}\left(a_{2}, a_{3}\right) \\
0 & 0 & \Lambda_{3}\left(a_{3}\right)
\end{array}\right]
$$

containing the contribution of each of the component to default risk.

We have deliberately avoided too much discussion of the stochastic default level, but there are many interesting questions about how to compute prices conditional on partial information about the default level, or when the default level is not independent of the stock price. (See [10] for a discussion of partial information in the standard first-passage default model.) Also, the question of how to model the default level $a_{t}$ has been left mostly open in this paper. This provides a significant source of extra flexibility. There are various interesting ways to think of the process $a_{t}$ as correlated to the stock price $S_{t}$ to form a more delicate model. The problem of developing pricing formulas in such cases is deferred to future work. 


\section{Proofs}

\subsection{Proof of Proposition 2.2}

As a warmup, consider the simple case of constant default level $a$. From (1),

$$
\forall \omega \in \Omega, t \geq 0, \quad \log \left(S_{t} / S_{t^{-}}\right)=Y_{t}-Y_{t^{-}}=\triangle Y_{t}
$$

The jump measure of $Y$ is the TPRM $X$ on $E=\mathbb{R} \backslash\{0\}$ with intensity $\lambda$. The default time $\tau$ is then

$$
\tau=\inf \left\{t>0: \triangle Y_{t} \leq a\right\}=\inf \left\{t>0: X_{t}(-\infty, a]>0\right\} .
$$

The process $N$ such that for $t \in \mathbb{R}_{+}, N_{t}=X_{t}(-\infty, a]$ is a homogeneous Poisson process of intensity $\Lambda(a)$. With these remarks, the probability of default is then

$$
\mathbb{P}(\tau \leq t)=1-\mathbb{P}\left(X_{t}(-\infty, a]=0\right)=1-e^{-t \Lambda(a)} .
$$

This simple argument extends to the case of non-constant deterministic default level $a_{t}$ by means of non-stationary Poisson processes.

Definition 7.1 ([7], Non-stationary Poisson process). A non-stationary Poisson process $M$ with intensity $(\lambda(t))_{t \geq 0}$, where $\lambda$ is a measurable deterministic function of time, is a counting process with independent increments such that for all $0 \leq s \leq t$, the increment $M_{t}-M_{s}$ has a Poisson distribution with parameter $\int_{s}^{t} \lambda_{u} d u$.

Notation: $I(z, A)$ denotes the indicator function with value 1 if $z \in A$ and 0 otherwise.

Proposition 7.2. Let $a: \mathbb{R}_{+} \rightarrow(-\infty, 0)$ be a measurable deterministic function bounded below zero, and $X$ a TPRM on $E=\mathbb{R} \backslash\{0\}$ adapted to $\left(\mathcal{F}_{t}\right)_{t \geq 0}$ with intensity measure $\lambda$. Define the process $N$ such that

$$
\forall \omega \in \Omega, t \geq 0, \quad N_{t}(\omega)=\int_{[0, t] \times E} I\left(z,\left(-\infty, a_{s}\right]\right) X(\omega, d s \times d z) .
$$

Then $N$ is a non-stationary Poisson process with intensity $\left(\Lambda\left(a_{t}\right)\right)_{t \geq 0}$.

Proof: Since $a$ is bounded below zero, say $a_{t} \leq-\epsilon$ for some $\epsilon>0$, and $\lambda$ is a Lévy measure, $\lambda\left(-\infty, a_{t}\right]=\Lambda\left(a_{t}\right)$ is uniformly bounded in $t$ by $\lambda(-\infty,-\epsilon]<\infty$.

The mapping $(s, z) \rightarrow G(s, z)=I\left(z,\left(-\infty, a_{s}\right]\right)$ from $\mathbb{R}_{+} \times \mathbb{R} \rightarrow \mathbb{R}_{+}$is deterministic, and as such $\mathcal{F}$-predictable, hence $N$ is adapted.

To see that $N$ is almost surely right continuous, note first that $N_{t}(\omega)$ is monotone increasing in $t$ for each $\omega$. Let $t_{n}$ be a sequence of times converging to $t_{0}$ from above. $N_{t_{n}}$ is then monotone decreasing and so $N_{t_{n}}-N_{t_{0}}$ converges pointwise to a non-negative random variable L. Moreover,

$$
\begin{aligned}
N_{t_{n}}-N_{t_{0}} & =\int_{\left(t_{0}, t_{n}\right] \times E} G(s, z) X(\omega, d s \times d z) \\
& \leq \int_{\left(t_{0}, t_{n}\right] \times(-\infty,-\epsilon]} X(\omega, d s \times d z)
\end{aligned}
$$

and so $\mathbb{E}\left[N_{t_{n}}-N_{t_{0}}\right] \leq\left(t_{n}-t_{0}\right) \lambda(-\infty,-\epsilon)$ converges to zero. By the dominated convergence theorem, $\mathbb{E}[L]=0$, so $L=0$ almost surely. 
Since $X$ is a Poisson random measure and $G$ takes only values 0 or $1, N$ jumps by one almost surely, and has independent increments by the independence property of $X$. Hence $N$ is a counting process with independent increments.

It remains to show that the increment $N_{t}-N_{t^{\prime}}$ is Poisson distributed with parameter $\int_{t^{\prime}}^{t} \Lambda\left(a_{s}\right) d s ;$ it suffices to compute the Laplace transform as

$$
\mathbb{E} e^{-\beta\left(N_{t}-N_{t^{\prime}}\right)}=\exp \left\{-\left(1-e^{-\beta}\right) \int_{t^{\prime}}^{t} \Lambda\left(a_{s}\right) d s\right\}
$$

Since $X$ is a Poisson random measure with intensity $L e b \times \lambda$, where $L e b$ is the Lebesgue measure, for any measurable function $f:[0, \infty) \times E \rightarrow \mathbb{R}_{+}$, we have the standard formula ([7], thm VI.2.9)

$$
\mathbb{E} e^{-X[f]}=\exp \left\{-(L e b \times \lambda)\left[1-e^{-f}\right]\right\} .
$$

Setting $f(s, z)=\beta I\left(s,\left[t^{\prime}, t\right]\right) I\left(z,\left(-\infty, a_{s}\right]\right)$, we have

$$
\begin{aligned}
\mathbb{E} e^{-\beta\left(N_{t}-N_{t^{\prime}}\right)} & =\mathbb{E} e^{-X[f]} \\
& =\exp \left\{-(\text { Leb } \times \lambda)\left[1-e^{-\beta I\left(s,\left[t^{\prime}, t\right]\right) I\left(z,\left(-\infty, a_{s}\right]\right)}\right]\right\} \\
& =\exp \left\{-\int_{t^{\prime}}^{t} \int_{-\infty}^{a_{s}} 1-e^{-\beta} d \lambda d s\right\} \\
& =\exp \left\{-\left(1-e^{-\beta}\right) \int_{t^{\prime}}^{t} \Lambda\left(a_{s}\right) d s\right\} .
\end{aligned}
$$

Now our next step is to prove Corollary 2.3.

The default time $\tau$ is the time of the first jump of $Y$ below the varying level $a_{t}$. Define the process $N$ such that

$$
\forall \omega \in \Omega, t \geq 0, \quad N_{t}(\omega)=\int_{[0, t] \times E} I\left(z,\left(-\infty, a_{s}\right]\right) X(\omega, d s \times d z) .
$$

$N$ counts the number of jumps of $Y_{s}$ falling in $\left(-\infty, a_{s}\right]$ over the times $s$, for $s \in[0, t]$. Prop. 7.2 shows that $N$ is non-stationary Poisson with intensity $\left(\Lambda\left(a_{t}\right)\right)_{t \geq 0}$. Therefore, the survival probability is

$$
\mathbb{P}(\tau>t)=\mathbb{P}\left(N_{t}=0\right)=\exp \left\{-\int_{0}^{t} \Lambda\left(a_{s}\right) d s\right\} .
$$

To compute the local default rate, note first that

$$
\begin{aligned}
\mathbb{P}(\tau \leq t+h \mid \tau>t) & =\frac{\mathbb{P}\left(\left[N_{t+h}>0\right] \wedge\left[N_{t}=0\right]\right)}{\mathbb{P}\left(N_{t}=0\right)} \\
& =\frac{\mathbb{P}\left(N_{t+h}-N_{t}>0\right) \mathbb{P}\left(N_{t}=0\right)}{\mathbb{P}\left(N_{t}=0\right)} \\
& =\mathbb{P}\left(N_{t+h}-N_{t}>0\right)=1-\exp \left\{-\int_{t}^{t+h} \Lambda\left(a_{s}\right) d s\right\} .
\end{aligned}
$$

If we write $F(s)=\Lambda\left(a_{s}\right)=\int_{-\infty}^{a_{s}} \lambda(d w)$, then $F$ is bounded and measurable, hence integrable on bounded intervals, so

$$
\frac{\mathbb{P}(\tau \leq t+h \mid \tau>t)}{h}=\frac{\int_{t}^{t+h} F(s) d s+o(h)}{h}
$$


and the right hand side converges to $F(t)=\Lambda\left(a_{t}\right)$ for almost every $t$ by the Fundamental Theorem of Calculus.

Additionally, if $a_{t}$ is right continuous and $\lambda$ has no atoms, then $F(s)$ is right continuous and the limit is equal to $F(t)$ for all $t$.

To complete the proof for the case of stochastic default level, let $\mathcal{F}_{t}^{a} \subset \mathcal{F}_{t}$ denote the filtration generated by $a_{t}$. By the tower property of conditional expectation,

$$
\mathbb{P}(\tau>t)=\mathbb{E}\left[\mathbb{P}\left(\tau>t \mid \mathcal{F}_{t}^{a}\right)\right]
$$

Because of independence, conditioning on $\mathcal{F}^{a}$ doesn't affect the distribution of $Y$, so the computations in the previous proof carry over for each fixed path of $a_{t}$. Therefore

$$
\mathbb{P}(\tau>t)=\mathbb{E}\left[\mathbb{P}\left(N_{t}=0 \mid \mathcal{F}_{t}^{a}\right)\right]=\mathbb{E}\left[\exp \left\{-\int_{0}^{t} \Lambda\left(a_{s}\right) d s\right\}\right] .
$$

The argument for the Hazard rate is similar with conditioning on $\mathcal{F}_{t+h}^{a}$, since $F$ is uniformly bounded, with the use of the Dominated Convergence Theorem.

$$
\begin{aligned}
\mathbb{P}(\tau \leq t+h \mid \tau>t) & =\mathbb{E}\left[1_{\{\tau \leq t+h\}} \mid \tau>t\right] \\
& =\mathbb{E}\left[\mathbb{E}\left[1_{\{\tau \leq t+h\}} \mid \tau>t, \mathcal{F}_{t+h}^{a}\right] \mid \tau>t\right] \\
& =\mathbb{E}\left[P\left(N_{t+h}-N_{t}=0 \mid \mathcal{F}_{t+h}^{a}\right) \mid \tau>t\right]
\end{aligned}
$$

where

$$
N_{t}(\omega)=\int_{[0, t] \times E} I\left(z,\left(-\infty, a_{s}\right]\right) X(\omega, d s \times d z)
$$

is a Cox process with intensity $\Lambda\left(a_{t}\right)$ (see [7, VI.6.2]), and conditional on $a_{t}$ is a non-stationary Poisson process. Therefore

$$
\mathbb{P}(\tau \leq t+h \mid \tau>t)=\mathbb{E}\left[1-\exp \left\{-\int_{t}^{t+h} \Lambda\left(a_{s}\right) d s\right\} \mid \tau>t\right] .
$$

Dividing by $h$ and taking the limit as $h \rightarrow 0$, we can pass the limit through the expectation since $\Lambda\left(a_{s}\right)$ is uniformly bounded, and the same argument as before gives us

$$
H_{t}=\mathbb{E}\left[\Lambda\left(a_{t}\right) \mid \tau>t\right]
$$

for a.e. $t$.

It remains to show that $\mathbb{E}\left[\Lambda\left(a_{t}\right) \mid \tau>t\right]=\frac{\mathbb{E}\left[\Lambda\left(a_{t}\right) e^{-\int_{0}^{t} \Lambda\left(a_{s}\right) d s}\right]}{\mathbb{E}\left[e^{-\int_{0}^{t} \Lambda\left(a_{s}\right) d s}\right]}$.

Following [3, Lemma 2.2.1, page 24] or [4, Sec 4.1], let $\left\{\mathcal{H}_{t}\right\}$ be the smallest filtration that makes $\tau$ a stopping time. It is observed that any integrable $\mathcal{H}_{t}$ measurable random variable $Y$ is of the form

$$
Y=h(\tau \wedge t)=h(\tau) 1_{\tau \leq t}+h(t) 1_{\tau>t}
$$

for some Borel function $h$.

Now let $X=\Lambda\left(a_{t}\right)$, an integrable $\mathcal{F}$-measurable random variable.

Since $\mathbb{E}\left[X \mid \mathcal{H}_{t}\right]$ is $\mathcal{H}_{t}$ measurable, we have

$$
\mathbb{E}\left[X \mid \mathcal{H}_{t}\right]=h(\tau) 1_{\tau \leq t}+h(t) 1_{\tau>t}
$$


for some Borel $h$. Multiplying both sides by $1_{\tau>t}$ and taking expectations gives

$$
\mathbb{E}\left[1_{\tau>t} \mathbb{E}\left[X \mid \mathcal{H}_{t}\right]\right]=h(t) \mathbb{E}\left[1_{\tau>t}\right]=h(t) \mathbb{P}(\tau>t) .
$$

Therefore

$$
h(t)=\mathbb{E}\left[X 1_{\tau>t}\right] / \mathbb{P}(\tau>t) .
$$

So, from equation (88),

$$
\mathbb{E}[X \mid \tau>t]=h(t)=\mathbb{E}\left[X 1_{\tau>t}\right] / \mathbb{P}(\tau>t)
$$

That is,

$$
H_{t}=\mathbb{E}\left[\Lambda\left(a_{t}\right) \mid \tau>t\right]=h(t)=\mathbb{E}\left[\Lambda\left(a_{t}\right) 1_{\tau>t}\right] / \mathbb{P}(\tau>t)
$$

Furthermore, using the tower property and conditioning on the filtration $\left(\mathcal{F}_{t}^{a}\right)_{t \geq 0}$ generated by the process $\left\{a_{t}\right\}$, we have

$$
\begin{aligned}
\mathbb{E}\left[\Lambda\left(a_{t}\right) 1_{\tau>t}\right] & =\mathbb{E}\left[\mathbb{E}\left[\Lambda\left(a_{t}\right) 1_{\tau>t} \mid \mathcal{F}_{t}^{a}\right]\right] \\
& =\mathbb{E}\left[\Lambda\left(a_{t}\right) \mathbb{E}\left[1_{\tau>t} \mid \mathcal{F}_{t}^{a}\right]\right] \\
& \left.=\mathbb{E}\left[\Lambda\left(a_{t}\right) \mathbb{P}\left(\tau>t \mid \mathcal{F}_{t}^{a}\right]\right)\right] \\
& \left.=\mathbb{E}\left[\Lambda\left(a_{t}\right) \mathbb{P}\left(N_{t}=0 \mid \mathcal{F}_{t}^{a}\right]\right)\right] \\
& =\mathbb{E}\left[\Lambda\left(a_{t}\right) e^{-\int_{0}^{t} \Lambda\left(a_{s}\right) d s}\right]
\end{aligned}
$$

Combining this with (86) yields

$$
H_{t}=\mathbb{E}\left[\Lambda\left(a_{t}\right) 1_{\tau>t}\right] / \mathbb{P}(\tau>t)=\frac{\mathbb{E}\left[\Lambda\left(a_{t}\right) e^{-\int_{0}^{t} \Lambda\left(a_{s}\right) d s}\right]}{\mathbb{E}\left[e^{-\int_{0}^{t} \Lambda\left(a_{s}\right) d s}\right]} .
$$

\subsection{Theorem 3.7}

The proof of Theorem 3.7 is separated into the following two Propositions.

Proposition 7.3. Let $(N, M)$ be a two dimensional Poisson process with parameters $\lambda, \mu$. Then there exists $\rho \leq \min (\lambda, \mu)$ and three independent Poisson processes $N^{\perp}, M^{\perp}$, and $L$, with respective parameters $\lambda-\rho, \mu-\rho, \rho$, such that, for all $t \geq 0$,

$$
\begin{aligned}
& N_{t}=N_{t}^{\perp}+L_{t} \\
& M_{t}=M_{t}^{\perp}+L_{t} .
\end{aligned}
$$

In particular, unless $\rho=0$ and then $N=N^{\perp}$ is independent of $M=M^{\perp}$, and jumps of $L$ correspond to simultaneous jumps of $N$ and $M$.

Proof: Since $N$ and $M$ are Poisson, the jumps of the Lévy process $(N, M)$ are limited to the cases $\left(\Delta N_{t}, \Delta M_{t}\right)=(1,0),(0,1)$, or $(1,1)$. By the Lévy-Itô decomposition [22], there is a TPRM $X$ on $E=[0, \infty)^{2} \backslash\{(0,0)\}$ with finite intensity $\pi$ supported on the three points $(1,0),(0,1),(1,1)$, and such that

$$
\left(N_{t}, M_{t}\right)=\int_{0}^{t} \int_{E} x X(d s \times d x) .
$$

July 2, 2016 
Recall our notation $X_{t}(A)=X([0, t] \times A)$. For convenience of notation, we temporarily write $A=\{(1,0)\}, B=\{(0,1)\}, C=\{(1,1)\}$ in $E$.

The integral above can then be written

$$
\left(N_{t}, M_{t}\right)=(1,0) X_{t}(A)+(0,1) X_{t}(B)+(1,1) X_{t}(C) .
$$

Since $X$ is a Poisson random measure, $N_{t}^{\perp}=X_{t}(A), M_{t}^{\perp}=X_{t}(B)$, and $L_{t}=X_{t}(C)$ are Poisson processes with parameters $\pi(A), \pi(B)$, and $\pi(C)$, respectively, and are independent because $A, B, C$ are disjoint. Equation 99 says that

$$
\begin{aligned}
& N_{t}=N_{t}^{\perp}+L_{t} \\
& M_{t}=M_{t}^{\perp}+L_{t} .
\end{aligned}
$$

If we let $\rho=\pi(C)$, this means the parameter of $N^{\perp}$ must be $\lambda-\rho$, and the parameter of $M^{\perp}$ must be $\mu-\rho$. This completes the proof.

Proposition 7.4. Let $N^{\perp}, M^{\perp}$, and $L$ be three independent Poisson processes with parameters $\nu_{1}, \nu_{2}$, and $\rho$. If $M$ and $N$ are defined by

$$
\begin{aligned}
& N_{t}=N_{t}^{\perp}+L_{t} \\
& M_{t}=M_{t}^{\perp}+L_{t}
\end{aligned}
$$

then $N$ and $M$ are Poisson and the joint survival times of $N$ and $M$ are bivariate exponential $b \mathcal{E}\left(\nu_{1}+\rho, \nu_{2}+\rho, \rho\right)$.

Proof: The sum of two independent $\mathcal{F}$-Poisson processes is a Poisson process with respect to $\left(\mathcal{F}_{t}\right)_{t \geq 0}$, see for instance [14]. Since $N^{\perp}, M^{\perp}$, and $L$ are independent, $N$ and $M$ are Poisson processes by construction.

For any $u \geq 0$, we want to show that the joint survival times $\left(\tau_{u}, \gamma_{u}\right)$ have the bivariate exponential distribution $b \mathcal{E}\left(\nu_{1}+\rho, \nu_{2}+\rho, \rho\right)$, in other words, for $s, t \geq 0$,

$$
\mathbb{P}\left(N_{s+u}-N_{u}=0, M_{t+u}-M_{u}=0\right)=e^{-\nu_{1} s-\nu_{2} t-\rho \max (s, t)} .
$$

The left hand side of this equation is, by hypothesis,

$$
\mathbb{P}\left(N_{s+u}^{\perp}-N_{u}^{\perp}=0, L_{s+u}-L_{u}=0, M_{t+u}^{\perp}-M_{u}^{\perp}=0, L_{t+u}-L_{u}=0\right) .
$$

Since $N^{\perp}, M^{\perp}$, and $L$ are independent and have stationary and independent increments, we obtain

$$
\begin{aligned}
& \mathbb{P}\left(N_{s+u}^{\perp}-N_{u}^{\perp}=0, L_{s+u}-L_{u}=0, M_{t+u}^{\perp}-M_{u}^{\perp}=0, L_{t+u}-L_{u}=0\right) \\
= & \mathbb{P}\left(N_{s+u}^{\perp}-N_{u}^{\perp}=0\right) \mathbb{P}\left(M_{t+u}^{\perp}-M_{u}^{\perp}=0\right) \mathbb{P}\left(L_{s+u}-L_{u}=0, L_{t+u}-L_{u}=0\right) \\
= & \mathbb{P}\left(N_{s}^{\perp}=0\right) \mathbb{P}\left(M_{t}^{\perp}=0\right) \mathbb{P}\left(L_{\max (s, t)+u}-L_{u}=0\right) \\
= & e^{-\nu_{1} s} e^{-\nu_{2} t} e^{-\rho \max (s, t)}
\end{aligned}
$$

\subsection{Proposition 3.8}

Proof: Since $(N, M)$ is a Lévy process, we have the usual decomposition $N=N^{\perp}+L, M=$ $M^{\perp}+L$, where $N^{\perp}, M^{\perp}, L$ are independent Poisson with parameters $\lambda-\rho, \mu-\rho, \rho$.

Let $u, v$ be possibly different times, and consider the distribution of the survival times $\left(\tau_{u}, \gamma_{v}\right)$.

July 2, 2016 


$$
\begin{aligned}
& P\left(\tau_{u}>s, \gamma_{v}>t\right)=P\left(N_{s+u}-N_{u}=0, M_{t+v}-M_{t}=0\right) \\
= & P\left(N_{s+u}^{\perp}-N_{u}^{\perp}=0, L_{s+u}-L_{u}=0, M_{t+v}^{\perp}-M_{v}^{\perp}=0, L_{t+v}-L_{v}=0\right) \\
= & P\left(N_{s+u}^{\perp}-N_{u}^{\perp}=0\right) P\left(M_{t+v}^{\perp}-M_{v}^{\perp}=0\right) P\left(L_{s+u}-L_{u}=0, L_{t+v}-L_{v}=0\right) \\
= & e^{-(\lambda-\rho) s} e^{-(\mu-\rho) t} P\left(L_{s+u}-L_{u}=0, L_{t+v}-L_{v}=0\right)
\end{aligned}
$$

We now claim $P\left(L_{s+u}-L_{u}=0, L_{t+v}-L_{v}=0\right)=e^{-\rho \Theta(s, t, u-v)}$. To see this, consider the various cases in which $[u, s+u]$ and $[v, t+v]$ can overlap, and use the stationary and independent increments properties of $L$.

\subsection{Theorem 3.15}

\section{Proof:}

Part (i) is Prop. 3.10. For part (ii), denote $N^{x}$ and $M^{y}$ the tail processes of $N$ and $M$ at the level $x$ and $y$, respectively, $x, y \in \mathbb{R} \backslash\{0\}$. $N^{x}$ and $M^{y}$ are Poisson processes with parameters $\Lambda(x)$ and $\Gamma(y)$ respectively. Since $(N, M)$ is a two dimensional Lévy process, $\left(N^{x}, M^{y}\right)$ is a two dimensional Poisson process, and we proceed as in the proof of Prop. 7.3.

By the Lévy-Itô decomposition, there is a TPRM $X^{x, y}$ on $E=[0, \infty)^{2} \backslash\{(0,0)\}$ with finite intensity $\pi^{x, y}$ supported on the three points $(1,0),(0,1),(1,1)$, and such that

$$
\begin{aligned}
\left(N_{t}^{x}, M_{t}^{y}\right) & =\int_{0}^{t} \int_{E} z X^{x, y}(d s \times d z) \\
& =(1,0) X_{t}^{x, y}(\{(1,0)\})+(0,1) X_{t}^{x, y}(\{(0,1)\})+(1,1) X_{t}^{x, y}(\{(1,1)\}) .
\end{aligned}
$$

If we denote the three independent Poisson processes as $N_{t}^{\perp}=X_{t}^{x, y}(\{(1,0)\}), M_{t}^{\perp}=$ $X_{t}^{x, y}(\{(0,1)\})$, and $L_{t}=X_{t}^{x, y}(\{(1,1)\})$, then the decomposition (103) satisfies the hypotheses of Proposition 7.4, and hence the joint survival times are bivariate exponential. Since the parameters of $N_{t}^{x}$ and $M_{t}^{y}$ are $\Lambda(x)$ and $\Gamma(y)$, respectively, it only remains to determine the parameter $\rho(x, y)$ of $L$.

Recall that if $P$ is any Poisson process, its parameter is given by $\mathbb{E}\left(P_{1}\right)$. So

$$
\left.\rho(x, y)=\mathbb{E}\left(L_{1}\right)=\mathbb{E} X_{1}^{x, y}(\{1,1)\}\right) .
$$

The number of joint jumps of $\left(N^{x}, M^{y}\right)$ corresponds to the number of jumps of $(N, M)$ of amplitude jointly larger than $(x, y)$. Therefore

$$
\rho(x, y)=\mathbb{E} X_{1}(\mathcal{I}(x) \times \mathcal{I}(y))=\Pi(x, y)
$$

and the proof of (ii) is complete.

Part (iii) follows from Theorem 3.7 and part (iv) from Theorem 3.14.

\subsection{Proof of Proposition 4.1}

As a warmup, first consider the easiest case of constant default levels $a_{1}, a_{2}$. With (39), an equivalent definition for the default times (41) is

$$
\tau_{i}=\inf \left\{t>0: \triangle Y_{t}^{i} \in \mathcal{I}\left(a_{i}\right)\right\}, \quad i=1,2 .
$$

Clearly, $\tau_{1}$ and $\tau_{2}$ are the joint survival times of $Y^{1}$ and $Y^{2}$ at the level $a_{1}$ and $a_{2}$ respectively. Since the process $Y=\left(Y_{1}, Y_{2}\right)$ is a Lévy process, Prop. 3.10 shows that $\tau_{1}$ and $\tau_{2}$ satisfy 
the two dimensional memoryless property. Thm. 3.15 shows that $\tau_{1}$ and $\tau_{2}$ are exponential random variables with parameters $\Lambda\left(a_{1}\right)$ and $\Gamma\left(a_{2}\right)$, and that there exists a parameter $\rho \in$ $\left[0, \min \left(\Lambda\left(a_{1}\right), \Gamma\left(a_{2}\right)\right)\right]$ such that

$$
\forall s, t \geq 0, \quad \mathbb{P}\left(\tau_{1}>s, \tau_{2}>t\right)=C_{\rho}^{\lambda, \mu}\left(e^{-\Lambda\left(a_{1}\right) s}, e^{-\Gamma\left(a_{2}\right) t}\right),
$$

where $C_{\rho}^{\lambda, \mu}$ is the 3-parameter Marshall-Olkin copula 3.3 with

$$
\lambda=\Lambda\left(a_{1}\right), \quad \text { and } \quad \mu=\Gamma\left(a_{2}\right) .
$$

Furthermore, Theorem 3.15(iv) implies, since $\operatorname{sgn}\left(a_{1} a_{2}\right)=1$, that there exists a Lévy copula $\rho(\cdot, \cdot)$ such that

$$
\rho=\Pi\left(a_{1}, a_{2}\right)=\pi\left(\mathcal{I}\left(a_{1}\right) \times \mathcal{I}\left(a_{2}\right)\right)=\rho\left(\bar{\Lambda}\left(a_{1}\right), \bar{\Gamma}\left(a_{2}\right)\right) .
$$

The next step is to prove Corollary 4.2. The proof relies on a slight extension of Prop. 7.2 to sets of $\mathbb{R}^{2}$. For convenience of notation we write $B(\epsilon)$ for the disk of radius $\epsilon$, i.e. for $\epsilon>0, B(\epsilon)=\left\{x \in \mathbb{R}^{2}:|x| \leq \epsilon\right\}$, and denote $B(\epsilon)^{c}$ its complement.

Definition 7.5. Let $(E, \mathcal{B}(E))$ be a measurable space. We say that the function $B: \mathbb{R}_{+} \rightarrow$ $\mathcal{B}(E)$ is a measurable set function in $E$ if the mapping $I: \mathbb{R}_{+} \times E \rightarrow \mathbb{R}$, defined by $I(s, z)=$ $I\left(z, B_{s}\right)$, is measurable on $\mathbb{R}_{+} \times E$.

Proposition 7.6. Let $E=\mathbb{R}^{2} \backslash\{(0,0)\}$ with $\mathcal{B}(E)$ the Lebesgue measurable subsets of $E$. Let $B: \mathbb{R}_{+} \rightarrow \mathcal{B}(E)$ be a measurable set function in $E$ that is bounded away from 0 , i.e. $\exists \epsilon>0, B_{t} \in B(\epsilon)^{c}, \forall t \geq 0$. Let $X$ be a TPRM on $E$ adapted to $\mathcal{F}_{t}$ with intensity $\pi$, where $\pi$ is a Lévy measure. Define the process $N$ such that

$$
\forall \omega \in \Omega, t \geq 0, \quad N_{t}(\omega)=\int_{[0, t] \times E} I\left(z, B_{s}\right) X(\omega, d s \times d z) .
$$

Then $N$ is a non-stationary Poisson process with intensity $\left(\pi\left(B_{t}\right)\right)_{t \geq 0}$.

Proof: The mapping $(s, z) \rightarrow I\left(z, B_{s}\right)=1$ if $z \in B_{s}$, 0 otherwise, is deterministic and therefore $\mathcal{F}$-predictable, so $N$ is adapted. Furthermore, $N$ jumps by 1 almost surely and has independent increments by construction from $X$.

Since $B_{t} \subset B_{\epsilon}^{c}$ for some $\epsilon>0$, and $\pi$ is a Lévy measure, $\pi\left(B_{t}\right) \leq \pi\left(B(\epsilon)^{c}\right)<\infty$. As before, since $N_{t}$ is increasing, given a sequence of times $t_{n}$ converging to $t$ from above, the random variables $L_{n}=N_{t_{n}}-N_{t}$ are monotone decreasing and bounded below by 0 . Thus $L_{n}$ converges to a non-negative random variable $L$. Furthermore,

$$
\begin{aligned}
L_{n} & =\int_{\left(t, t_{n}\right] \times E} I\left(z, B_{s}\right) X(\omega, d s \times d z) \leq \int_{\left(t, t_{n}\right] \times E} I\left(z, B(\epsilon)^{c}\right) X(\omega, d s \times d z) \\
& \leq \int_{\left(t, t_{n}\right] \times B(\epsilon)^{c}} X(\omega, d s \times d z) .
\end{aligned}
$$

Therefore, $\mathbb{E}\left[L_{n}\right] \leq\left(t_{n}-t\right) \pi\left(B(\epsilon)^{c}\right)<\infty$ converges to 0 . The dominated convergence theorem implies that $\mathbb{E}[L]=0$, hence $L=0$ almost surely, so $N$ is a right continuous process. 
To show that $N$ is a non-stationary Poisson process with intensity $\pi\left(B_{t}\right)$, it remains to show that, for $0 \leq s \leq t$, the increments $N_{t}-N_{s}$ of $N$ have the Poisson distribution with parameter $\int_{s}^{t} \pi\left(B_{u}\right) d u$. It is sufficient to compute its Laplace transform, $0 \leq s \leq t, \beta \geq 0$,

$$
\mathbb{E} \exp -\beta\left(N_{t}-N_{s}\right)=\exp \left\{-\left(1-e^{-\beta}\right) \int_{s}^{t} \pi\left(B_{u}\right) d u\right\}
$$

The result above is obtained by letting $f:[0, \infty) \times E \rightarrow \mathbb{R}_{+}$be the function defined as $f(u, z)=\beta I(u,[s, t]) I\left(z, B_{u}\right)$. Since $f$ is measurable, we can use the formula (83)

$$
\mathbb{E} e^{-X[f]}=\exp \left\{-(L e b \times \pi)\left[1-e^{-f}\right]\right\} .
$$

Eq. (110) follows from the same computations as for the proof of Prop. 7.2.

Lemma 7.7. Let $Y$ be a two dimensional Lévy process with Lévy measure $\pi$ and tail integrals $\Lambda$ and $\Gamma$. Let $a, b \in(-\infty, 0)$ and define the set $A=\mathbb{R}^{2} \backslash(a, \infty) \times(b, \infty)$. Then there exists a Lévy copula $\rho(\cdot, \cdot)$ such that

$$
\pi(A)=\Lambda(a)+\Gamma(b)-\rho(\bar{\Lambda}(a), \bar{\Gamma}(b)) .
$$

Proof: For convenience of notation we define the sets

$$
B=(-\infty, a) \times \mathbb{R}, \quad C=(-\infty, a) \times(-\infty, b), \quad D=\mathbb{R} \times(-\infty, b) .
$$

The set $A$ can be decomposed in the following disjoint sets

$$
A=(B \backslash C) \cup(D \backslash C) \cup C .
$$

Considering the margins of $\pi$, we have $\pi(B)=\Lambda(a)$ and $\pi(D)=\Gamma(b)$, and since $\pi$ is a measure, this gives

$$
\pi(A)=\Lambda(a)+\Gamma(b)-\pi((-\infty, a) \times(-\infty, b)) .
$$

We conclude with an application of Thm. 3.14, which gives the existence of a Lévy copula $\rho$ such that

$$
\pi((-\infty, a) \times(-\infty, b))=\rho(\bar{\Lambda}(a), \bar{\Gamma}(b)) .
$$

This concludes the proof of Lemma 7.7.

Continuing the proof of Corollary 4.2, define two Borel measurable functions $A, B$ : $[0, \infty) \rightarrow \mathcal{B}(E)$ by

$$
A_{t}=\mathbb{R}^{2} \backslash\left(\left(a_{t}^{1}, \infty\right) \times\left(a_{t}^{2}, \infty\right)\right), \quad B_{t}=\left(-\infty, a_{t}^{1}\right] \times \mathbb{R} .
$$

Since $a^{1}$ and $a^{2}$ are bounded below 0 , there exists $\epsilon>0$ such that $A_{t}, B_{t} \subset B(\epsilon)^{c}$ for all $t \geq 0$. Define the processes $P$ and $Q$ as

$$
P_{t}=\int_{[0, t] \times E} I\left(z, A_{s}\right) X(\omega, d s \times d z), \quad Q_{t}=\int_{[0, t] \times E} I\left(z, B_{s}\right) X(\omega, d s \times d z) .
$$

By Prop. 7.6, $P$ and $Q$ are non-stationary Poisson processes with intensity $\pi\left(A_{t}\right)$ and $\pi\left(B_{t}\right)$ respectively. $P$ counts the number of jumps of $Y=\left(Y^{1}, Y^{2}\right)$ falling in $A_{t}$ and $Q$ counts the number of jumps of $Y$ falling into $B_{t}$. 
Without loss of generality, assume $0 \leq t \leq s$. We have

$$
\begin{aligned}
{\left[\tau_{1}>s, \tau_{2}>t\right] } & =\left[\triangle Y_{u}^{1} \notin\left(-\infty, a_{u}^{1}\right]: u \leq s, \triangle Y_{u}^{2} \notin\left(-\infty, a_{u}^{2}\right]: u \leq t\right] \\
& =\left[\left(\triangle Y_{u}^{1}, \triangle Y_{u}^{2}\right) \notin A_{u}: u \leq t,\left(\triangle Y_{u}^{1}, \triangle Y_{u}^{2}\right) \notin B_{u}: t<u \leq s\right] \\
& =\left[P_{t}-P_{0}=0, Q_{s}-Q_{t}=0\right] .
\end{aligned}
$$

Furthermore, the increments $P_{t}-P_{0}=0$ and $Q_{s}-Q_{t}$ are independent by construction since $X$ is a TPRM and the intervals $[0, t]$ and $[t, s]$ are disjoint. Then

$$
\begin{aligned}
\mathbb{P}\left(\tau_{1}>s, \tau_{2}>t\right) & =\mathbb{P}\left(P_{t}-P_{0}=0, Q_{s}-Q_{t}=0\right) \\
& =\mathbb{P}\left(P_{t}-P_{0}=0\right) \mathbb{P}\left(Q_{s}-Q_{t}=0\right) \\
& =\exp -\left\{\int_{0}^{t} \pi\left(A_{u}\right) d u+\int_{t}^{s} \pi\left(B_{u}\right) d u\right\} .
\end{aligned}
$$

Using Lemma 7.7,

$$
\pi\left(A_{u}\right)=\Lambda\left(a_{u}^{1}\right)+\Gamma\left(a_{u}^{2}\right)-\rho\left(\bar{\Lambda}\left(a_{u}^{1}\right), \bar{\Gamma}\left(a_{u}^{2}\right)\right),
$$

and $\pi\left(B_{u}\right)=\lambda\left(-\infty, a_{u}^{1}\right]=\Lambda\left(a_{u}^{1}\right)$. Therefore

$$
\begin{aligned}
\mathbb{P}\left(\tau_{1}>s, \tau_{2}>t\right) & =\exp -\left\{\int_{0}^{t} \Lambda\left(a_{u}^{1}\right)+\Gamma\left(a_{u}^{2}\right)-\rho\left(\bar{\Lambda}\left(a_{u}^{1}\right), \bar{\Gamma}\left(a_{u}^{2}\right)\right) d u+\int_{t}^{s} \Lambda\left(a_{u}^{1}\right) d u\right\} \\
& =\exp -\left\{\int_{0}^{s} \Lambda\left(a_{u}^{1}\right)+\int_{0}^{t} \Gamma\left(a_{u}^{2}\right)-\int_{0}^{t} \rho\left(\bar{\Lambda}\left(a_{u}^{1}\right), \bar{\Gamma}\left(a_{u}^{2}\right)\right) d u\right\} .
\end{aligned}
$$

The case where $0 \leq s<t$ is symmetric and gives

$$
\mathbb{P}\left(\tau_{1}>s, \tau_{2}>t\right)=\exp -\left\{\int_{0}^{s} \Lambda\left(a_{u}^{1}\right)+\int_{0}^{t} \Gamma\left(a_{u}^{2}\right)-\int_{0}^{s} \rho\left(\bar{\Lambda}\left(a_{u}^{1}\right), \bar{\Gamma}\left(a_{u}^{2}\right)\right) d u\right\},
$$

The extension of Corollary 4.2 proceeds as in the one-dimensional case. Because of the independence assumption, conditioning on $\left(a^{1}, a^{2}\right)$ doesn't affect the distribution of $Y$, so the arguments carry over via the tower property for each fixed path of the default levels.

\subsection{Proposition 4.4}

Proof: The proof is based on the little- $o$ property for stationary processes. Define three disjoint regions $B_{1}, B_{2}, B_{12} \subset \mathbb{R}^{2}$ of the plane as

$$
B_{1}=\left(-\infty, a_{1}\right] \times\left(a_{2}, 0\right), \quad B_{2}=\left(a_{1}, 0\right) \times\left(-\infty, a_{2}\right], \quad B_{1,2}=\left(-\infty, a_{1}\right] \times\left(-\infty, a_{2}\right] .
$$

Define the disjoint sets

$$
\begin{array}{lc}
E_{1}= & (t, t+h] \times B_{1} \\
E_{2}= & (t, t+h] \times B_{2} \\
E_{3}= & (t, t+h] \times B_{1,2} \\
E_{4}= & {[0, t] \times\left(B_{1} \cup B_{2} \cup B_{1,2}\right) .}
\end{array}
$$

Then we have the following four independent events:

$$
F_{i}=\left[X\left(E_{i}\right)>0\right], i=1,2,3 \text {, and } F_{4}=\left[X\left(E_{4}\right)=0\right] .
$$


From our definitions, $F_{1}$ is the event that there exists $s \in(t, t+h]$ such that $\left(\triangle Y_{s}^{1}, \triangle Y_{s}^{2}\right) \in$ $B_{1}, F_{2}$ is the event that there exists $u \in(t, t+h]$ such that $\left(\triangle Y_{u}^{1}, \triangle Y_{u}^{2}\right) \in B_{2}, F_{3}$ is the event that there exists $w \in(t, t+h]$ such that $\left(\triangle Y_{w}^{1}, \triangle Y_{w}^{2}\right) \in B_{1,2}$, and $F_{4}$ is the event that there are no jumps in $B_{1,2}$ up to time $t$.

With this notation, the event that neither firm defaults by time $t$ is

$$
\left[\tau_{1} \wedge \tau_{2}>t\right]=F_{4}
$$

and the event that both firms default between times $t$ and $t+h$ is

$$
\left[\tau_{1} \vee \tau_{2} \leq t+h, \tau_{1} \wedge \tau_{2}>t\right]=\left(\left(F_{1} \cap F_{2}\right) \cup F_{3}\right) \cap F_{4}
$$

We can then compute the conditional probability in the statement of the Proposition:

$$
\begin{aligned}
& \mathbb{P}\left(\tau_{1} \vee \tau_{2} \leq t+h \mid \tau_{1} \wedge \tau_{2}>t\right)=\frac{\mathbb{P}\left(\left(\left(F_{1} \cap F_{2}\right) \cup F_{3}\right) \cap F_{4}\right)}{\mathbb{P}\left(F_{4}\right)}=\mathbb{P}\left(\left(\left(F_{1} \cap F_{2}\right) \cup F_{3}\right)\right) \\
= & \mathbb{P}\left(X\left((t, t+h] \times B_{1}\right)>0\right) \mathbb{P}\left(X\left((t, t+h] \times B_{2}\right)>0\right)+\mathbb{P}\left(X\left((t, t+h] \times B_{1,2}>0\right)\right. \\
= & \mathbb{P}\left(X\left((t, t+h] \times B_{1}\right)=1\right) \mathbb{P}\left(X\left((t, t+h] \times B_{2}\right)=1\right)+\mathbb{P}\left(X\left((t, t+h] \times B_{1,2}=1\right)+o(h)\right. \\
= & \pi\left(B_{1}\right) \pi\left(B_{2}\right) h^{2} e^{-h\left(\pi\left(B_{1}\right)+\pi\left(B_{2}\right)\right)}+\pi\left(B_{1,2}\right) h e^{-h \pi\left(B_{1,2}\right)}+o(h) .
\end{aligned}
$$

Finally,

$$
\lim _{h \downarrow 0} \frac{\mathbb{P}\left(\tau_{1} \vee \tau_{2} \leq t+h \mid \tau_{1} \wedge \tau_{2}>t\right)}{h}=\pi\left(B_{1,2}\right)=\Pi\left(a_{1}, a_{2}\right),
$$

and we conclude with (108). 
A Structural Jump Threshold Framework

\section{References}

[1] David Applebaum, Lévy processes and stochastic calculus, vol. 93, Cambridge university press, 2004.

[2] J. Bertoin, Lévy processes, vol. 121, Cambridge university press, 1998.

[3] T.R. Bielecki, M. Jeanblanc, and M. Rutkowski, Credit risk, Lecture Notes of M. Jeanblanc, Lisbon, Portugal., (2006), pp. 1-239.

[4] T.R. Bielecki And M. Rutkowski, Credit risk: modeling, valuation and hedging, Springer, 2004.

[5] Fischer Black and John C. Cox, Valuing corporate securities: Some effects of bond indenture provisions, The Journal of Finance, 31 (1976), pp. pp. 351-367.

[6] Damiano Brigo And Aurélien Alfonsi, Credit default swaps calibration and option pricing with the ssrd stochastic intensity and interest-rate model, in Proceedings of the 6-th Columbia = JAFEE International Conference, Citeseer, 2003.

[7] Erhan Cinlar, Introduction to stochastic processes, Courier Dover Publications, 2013.

[8] R. Cont and P. Tankov, Financial modelling with jump processes, vol. 2, Chapman \& Hall/CRC, 2003.

[9] Pierre Garreau, Jump Dependence and Multidimensional Default Risk: A New Class of Structural Models with Stochastic Intensities, electronic dissertation, paper 8555, Florida State University, 2013.

[10] Kay Giesecke, Default and information, Journal of Economic Dynamics \& Control, 30 (2006), pp. 2281-2303.

[11] Robert A. Jarrow and Stuart M. Turnbull, Pricing derivatives on financial securities subject to credit risk, The Journal of Finance, 50 (1995), pp. pp. 53-85.

[12] RoBert A JARROW AND FAN YU, Counterparty risk and the pricing of defaultable securities, the Journal of Finance, 56 (2001), pp. 1765-1799.

[13] J. Kallsen and P. Tankov, Characterization of dependence of multidimensional lévy processes using lévy copulas, Journal of Multivariate Analysis, 97 (2006), pp. 1551-1572.

[14] John Frank Charles Kingman, Poisson processes, vol. 3, Oxford university press, 1992.

[15] S. G. Kou And Hui Wang, First passage times of a jump diffusion process, Advances in Applied Probability, 35 (2003), pp. pp. 504-531.

[16] A. Kyprianou, Introductory lectures on fluctuations of Lévy processes with applications, Springer, 2006.

[17] Dilip Madan And Wim Schoutens, Break on through to the single side, Journal of Credit Risk, 4 (2008), pp. 3-20.

[18] Benoit Mandelbrot, Sur certains prix spéculatifs: faits empiriques et modèle basé sur les processus stables additifs de paul lévy, Comptes Rendus, 254 (1962), pp. 3968-3970.

July 2, 2016 
[19] A.W. Marshall and I. Olkin, A multivariate exponential distribution, Journal of the American Statistical Association, 62 (1967), pp. 30-44.

[20] R.B. Nelsen, An introduction to copulas, Springer, 1999.

[21] L. C. G. Rogers, Evaluating first-passage probabilities for spectrally one-sided lévy processes, Journal of Applied Probability, 37 (2000), pp. pp. 1173-1180.

[22] K. Sato, Lévy processes and infinitely divisible distributions, Cambridge university press, 1999.

[23] P.J. SchönBucher, Credit derivatives pricing models: models, pricing and implementation, Wiley, 2003.

[24] W. Schoutens and J. Cariboni, Lévy processes in credit risk, vol. 458, Wiley, 2009.

[25] P. Tankov, Processus de Lévy en finance: problèmes inverses et modélisation de dépendance, $\mathrm{PhD}$ thesis, Ecole Polytechnique X, 2004. 